\title{
Gravity, Magnetic, and Physical Property Data in the Smoke Creek Desert Area, Northwest Nevada
}

By Janet E. Tilden, David A. Ponce, Jonathan M.G. Glen, Bruce A. Chuchel, Kira Tushman, and Alison Duvall

2006

Any use of trade, product, or firm names is for descriptive purposes only and does not imply endorsement by the U.S. Government

Open-File Report 2006-1197

U.S. Department of the Interior

U.S. Geological Survey 


\section{Contents}

Abstract

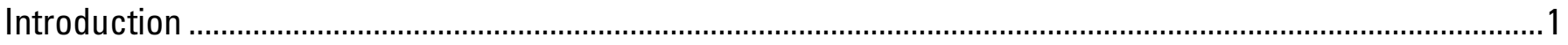

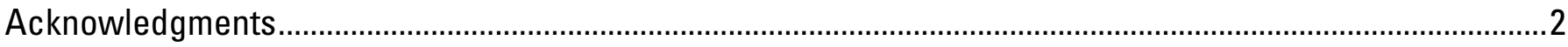

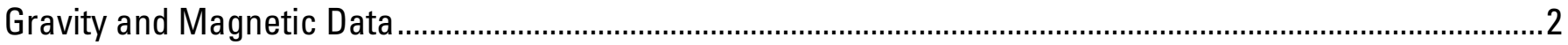

Gravity Methods

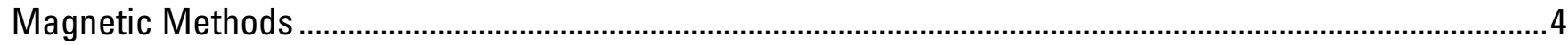

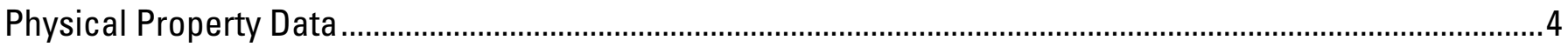

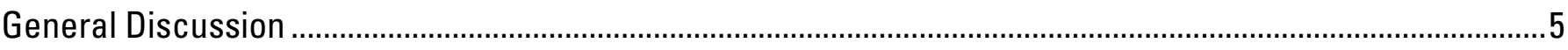

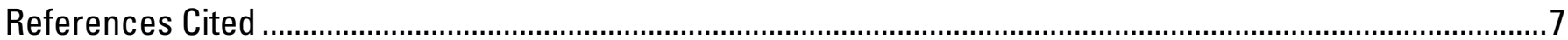

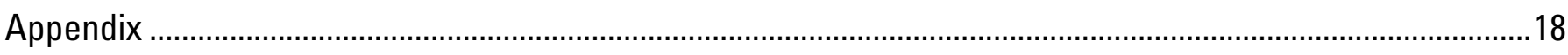

\section{Figures}

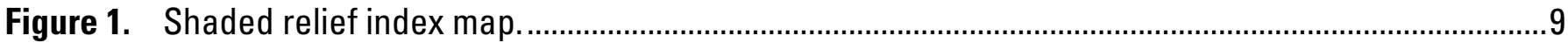

Figure 2. Simplified geologic map …………………………...........................................................

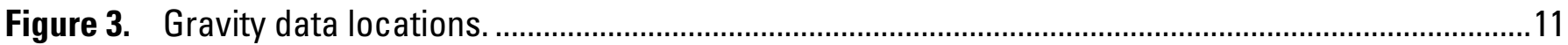

Figure 4. Truck-towed magnetometer survey lines ...............................................................................12

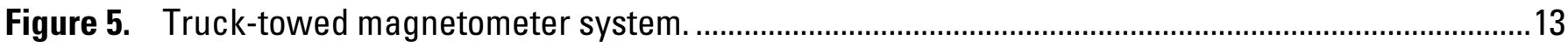

Figure 6. Flight line map for the detailed aeromagnetic survey ................................................................14

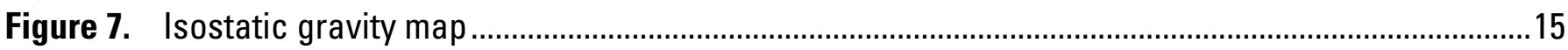

Figure 8. Truck-towed magnetometer ground magnetic map ...................................................................16

Figure 9. Detailed and regional aeromagnetic map ..................................................................................17

\section{Tables}

Table A1a. Physical property measurements of rock samples in the Smoke Creek Desert area....................18

Table A1b. Mean physical property data by rock type................................................................................21

Table A2. Principal facts of gravity stations in the Smoke Creek Desert area..................................................22 


\title{
Gravity, Magnetic, and Physical Property Data in the Smoke Creek Desert Area, Northwest Nevada
}

\author{
By Janet E. Tilden, David A. Ponce, Jonathan M.G. Glen, Bruce A Chuchel, Kira Tushman, and \\ Alison Duvall
}

\begin{abstract}
The Smoke Creek Desert, located approximately $100 \mathrm{~km}(60 \mathrm{mi})$ north of Reno near the California-Nevada border, is a large basin situated along the northernmost parts of the Walker Lane Belt (Stewart, 1988), a physiographic province defined by northwest-striking topographic features and strike-slip faulting. Because geologic framework studies play an important role in understanding the hydrology of the Smoke Creek Desert, a geologic and geophysical effort was begun to help determine basin geometry, infer structural features, and estimate depth to PreCenozoic rocks, or basement.

In May and June of 2004, and June of 2005, the U.S. Geological Survey (USGS) collected 587 new gravity stations, more than 160 line-kilometers (100 line-miles) of truck-towed magnetometer data, and 111 rock property samples in the Smoke Creek Desert and vicinity in northwest Nevada, as part of an effort to characterize its hydrogeologic framework. In the Smoke Creek Desert area, gravity highs occur over rocks of the Skedaddle Mountains, Fox Range, Granite Range, and over portions of Tertiary volcanic rocks in the Buffalo Hills. These gravity highs likely reflect basement rocks, either exposed at the surface or buried at shallow depths. The southern Smoke Creek Desert corresponds to a $25-\mathrm{mGal}$ isostatic gravity low, which corresponds with a basin depth of approximately $2 \mathrm{~km}$.

Magnetic highs are likely due to granitic, andesitic, and metavolcanic rocks, whereas magnetic lows are probably associated with less magnetic gneiss and metasedimentary rocks in the region. Three distinctive patterns of magnetic anomalies occur throughout the Smoke Creek Desert and Squaw Creek Valley, likely reflecting three different geological and structural settings.
\end{abstract}

\section{Introduction}

In May and June of 2004, and June of 2005, the U.S. Geological Survey (USGS) collected 587 new gravity stations, more than 160 line-kilometers (100 line-miles) of truck-towed magnetometer data, and 111 rock property samples in the Smoke Creek Desert and vicinity in northwest Nevada (fig. 1), as part of an effort to characterize its hydrogeologic framework. The Smoke Creek Desert, located approximately $100 \mathrm{~km}(60 \mathrm{mi})$ north of Reno near the CaliforniaNevada border (fig. 1), is a large basin situated along the northernmost parts of the Walker Lane Belt (Stewart, 1988), a physiographic province defined by northwest-striking topographic features and strike-slip faulting. Because geologic framework studies play an important role in understanding the hydrology of the Smoke Creek Desert, a geologic and geophysical effort was begun to help determine basin geometry, infer structural features, and estimate depth to PreCenozoic rocks, or basement. The study area is bounded by Mesozoic and Paleozoic metamorphic 
rocks and Cretaceous granitic rocks in the Fox and Granite Ranges and along the western margin of the Smoke Creek Desert (fig. 2). Tertiary basalts are mapped to the north, west, and south of the Smoke Creek Desert playa in the Buffalo Hills, Skedaddle Mountains, and Terraced Hills, respectively (fig. 2). Metamorphic, granitic, and volcanic rocks are important because they exhibit densities and magnetic susceptibilities higher than other Tertiary and Quaternary sedimentary rocks of the region, creating a distinguishable pattern of gravity and magnetic anomalies that reflect these properties.

\section{Acknowledgments}

We would like to thank Robert L. Morin of the U.S. Geological Survey for co-development of the truck-towed magnetometer system used in this study. Also, we would like to thank Bruce Chuchel, Alison Duvall, and Kira Tushman for their assistance in collecting the geophysical data. Thanks to Vicki Langenheim and Geoff Phelps for their review comments and suggestions. This study was funded by Sempre Energy.

\section{Gravity and Magnetic Data}

The gravity data in this report consist of 587 newly collected stations concentrated in the Smoke Creek Desert and the surrounding area (fig. 3). Of these, 108 stations were collected by helicopter in the surrounding mountain ranges and 251 closely-spaced gravity stations were collected along twelve transects that cross the Smoke Creek Desert playa a station spacing of about 400-m (1/4-mi). Additional gravity stations were primarily collected along existing roads within the Smoke Creek Desert area. The 251 closely-spaced gravity station locations correspond to transects along which truck-towed magnetometer data were also collected. All gravity data are tied to the primary base station RENO-A on the University of Nevada, Reno (UNR) campus at $39^{\circ}$ $32.30^{\prime} \mathrm{N}$ and $119^{\circ} 48.70^{\prime} \mathrm{W}$, with an observed gravity value of 979,674.69 milligals (mGal). New gravity stations were located between latitudes $40^{\circ} 10^{\prime}$ and $40^{\circ} 52^{\prime} \mathrm{N}$ and longitudes $119^{\circ} 15^{\prime}$ and $120^{\circ} 0^{\prime} \mathrm{W}$ and are on the Lovelock $1 \times 2$ degree (1:250,000-scale) quadrangle. These data were combined with pre-existing gravity data from the surrounding USGS quadrangles (Snyder and others, 1981; Ponce, 1997). Principle facts of these newly collected gravity stations can be found in the Appendix (table A2).

More than 160 line-kilometers (100 line-miles) of truck-towed magnetometer data were collected along nineteen transects located within the Smoke Creek Desert and one transect within Squaw Creek Valley (fig. 4). Magnetic data were collected using a cesium vapor magnetometer attached to an aluminum carriage connected to the vehicle by aluminum tubing and towed about 9 m (30 ft) behind the vehicle (fig. 5). Magnetometer and Geographic Positioning System (GPS) data were collected simultaneously at one-second intervals, which at an average speed of $30 \mathrm{mph}$, corresponds to approximately one measurement every $13.4 \mathrm{~m}(44 \mathrm{ft})$. A centrally located portable proton-precession base station magnetometer was used to record diurnal variation of the Earth's magnetic field during the truck-towed magnetometer surveys.

Aeromagnetic data were collected by Sander Geophysics Ltd., a geophysical service company based in Ottawa, Canada that specializes in high-precision airborne surveys (fig. 6). The airborne survey was flown at a flight-line spacing of $200 \mathrm{~m}$ (1/8 mi) over the Smoke Creek Desert and $400 \mathrm{~m}$ (1/4-mi) in the Squaw Creek Valley, at a nominal flight elevation above the ground of $150 \mathrm{~m}$, and with an east-west flight-line direction. North-south tie or control lines were flown at a flight-line spacing of 2,400 $\mathrm{m}$ (1.5 mi). Both aerial and ground based magnetometers used in the survey were pumped-cesium vapor magnetometers by Geometrics (Model G-822A), with a 
sensitivity of about $0.005 \mathrm{nT}$ (nanoteslas). A real-time differential GPS system was used for positional data and on-board navigation, capable of guiding the aircraft along a pre-flight path in three dimensions, with an accuracy of about $0.2 \mathrm{~m}(0.7 \mathrm{ft})$.

All gravity, ground magnetic, and aeromagnetic data collected for this survey can be downloaded from this website http://pubs.usgs.gov/of/2006/1197/. The files are ascii comma delimited and are formatted for easy insertion into a database. Each data file has an associated readme document with necessary metadata information.

\section{Gravity Methods}

All gravity data were reduced using standard gravity methods (Blakely, 1995) and include the following corrections: (a) the earth-tide correction, which corrects for tidal effects of the moon and sun; (b) instrument drift correction, which compensates for drift in the instrument's spring; (c) the latitude correction, which incorporates the variation of the Earth's gravity with latitude; (d) the free-air correction, which accounts for the variation in gravity due to elevation relative to sea level; (e) the Bouguer correction, which corrects for the attraction of material between the station and sea level; (f) the curvature correction, which corrects the Bouguer correction for the effect of the Earth's curvature; $(\mathrm{g})$ the terrain correction, which removes the effect of topography to a radial distance of $167 \mathrm{~km}(104 \mathrm{mi})$; and (h) the isostatic correction, which removes long-wavelength variations in the gravity field inversely related to topography.

Conversion of meter readings to gravity units was made using factory calibration constants as well as a secondary calibration factor determined by multiple gravity readings over the Mt. Hamilton calibration loop east of San Jose, California (Barnes and others, 1969). The gravity meters used in this survey, LaCoste and Romberg G614 and G17C, have secondary calibration factors of 1.00036 and 1.00078 respectively. Observed gravity values were based on a timedependent linear drift between successive base readings and were referenced to the International Gravity Standardization Net 1971 gravity datum (Morelli, 1974, p. 18). Free-air gravity anomalies were calculated using the Geodetic Reference System 1967 formula for theoretical gravity on the ellipsoid (International Union of Geodesy and Geophysics, 1971, p. 60) and Swick's formula (Swick, 1942, p. 65) for the free-air correction. Bouguer, curvature, and terrain corrections were added to the free-air anomaly to determine the complete Bouguer anomaly at a standard reduction density of $2.67 \mathrm{~g} / \mathrm{cm}^{3}$. Finally, a regional isostatic gravity field was removed from the Bouguer field assuming an Airy-Heiskanen model for isostatic compensation of topographic loads (Jachens and Roberts, 1981) with an assumed crustal thickness of $25 \mathrm{~km}$ (16 mi), a crustal density of 2.67 $\mathrm{g} / \mathrm{cm}^{3}$, and a density contrast across the base of the model of $0.4 \mathrm{~g} / \mathrm{cm}^{3}$. Gravity values are expressed in $\mathrm{mGal}$, a unit of acceleration or gravitational force per mass equal to $10^{-5} \mathrm{~m} / \mathrm{s}^{2}$. Gravity data were gridded at an interval of $400 \mathrm{~m}$ (1/4 mi) using a computer program (Webring, 1981) based on a minimum curvature algorithm by Briggs (1974) and displayed as a color-contoured map (fig. 7).

Station locations and elevations were obtained using Trimble ${ }^{\varpi}$ differential Global Positioning Systems (DGPS); 1) GeoExplorer CE handheld receiver and 2) Ag132 pole-mounted receiver. The GeoExplorer CE receiver uses Wide Area Augmentation System (WAAS) correction messages, which combined with base station post-processing results in sub-meter vertical accuracy. The Ag132 receiver has real-time differential correction capabilities using an Omnistar satellite system, resulting in sub-meter horizontal accuracy and approximately 1-2 m (3-6 ft) vertical accuracy.

Terrain corrections, which account for the variation of topography near a gravity station, were computed using a combination of manual and digital methods. Terrain corrections consist of a 
three-part process: the innermost or field terrain correction, inner-zone terrain correction, and outer-zone terrain correction. Field terrain corrections were estimated in the field and extend from the station to a radial distance of $68 \mathrm{~m}(223 \mathrm{ft})$, equivalent to Hayford and Bowie (1912) zone B. Inner-zone terrain corrections were estimated from Digital Elevation Models (DEMs) with 10-m or

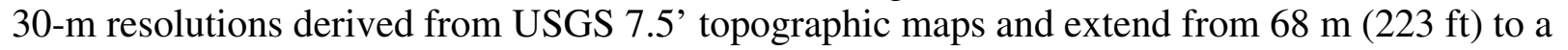
radial distance of $2 \mathrm{~km}$ (1.25 mi) (D. Plouff, USGS, unpublished software, 2005). Outer-zone terrain corrections, to a radial distance of $167 \mathrm{~km}$ (104 mi), were computed using a DEM derived from USGS 1:250,000-scale topographic maps and an automated procedure (Plouff, 1966; Plouff, 1977; Godson and Plouff, 1988). Digital terrain corrections are calculated by computing the gravity effect of each grid cell using the distance and difference in elevation of each grid cell from the gravity station.

\section{Magnetic Methods}

During field operations, towed-magnetic data were recorded and viewed in real-time using Geometrics ${ }^{\circledR}$ MagLog software. Raw magnetic data were downloaded and processed using MagMap2000 software, where magnetometer and GPS data were merged. The location of the magnetometer was recorded using the Trimble ${ }^{\circledR}$ non-magnetic Ag132 GPS receiver (described above) mounted on an aluminum frame attached to the magnetometer. Diurnal variations recorded by the centrally-located base station magnetometer were removed from the dataset and the magnetic data were filtered to remove cultural "noise" such as passing cars, culverts, fences, and power lines. Magnetic data were gridded and displayed as a color-contoured map (fig. 8).

Aeromagnetic data were reduced to total intensity magnetic field and include corrections for the diurnal variations of the Earth's magnetic field, despiking, leveling, and removal of a regional magnetic field of the Earth (IGRF) using the IGRF 2000 model (Langel, 1992). Aeromagnetic data were gridded at an interval of $50 \mathrm{~m}(165 \mathrm{ft})$ using a computer program (Webring, 1981) based on a minimum curvature algorithm by Briggs (1974) and displayed as a color-contoured map (fig. 9).

\section{Physical Property Data}

Density and magnetic properties of rock samples are used for gravity and magnetic modeling, as well as gravity inversion calculations. The 109 rock samples were collected in the mountains surrounding the Smoke Creek Desert (figure 6) for rock property analyses. Densities were determined using a precision Sartorius electronic balance. All rocks were weighed dry in air (Wa), saturated in water (Ww), and saturated with water in air (Ws). From these measurements, grain density, dry bulk density, and saturated bulk density were calculated using the following formulas:

$$
\begin{aligned}
& \text { Grain density }=\mathrm{Wa} /(\mathrm{Wa}-\mathrm{Ws}) \\
& \text { Dry bulk density }=\mathrm{Wa} /(\mathrm{Ws}-\mathrm{Ww}) \\
& \text { Saturated bulk density }=\mathrm{Ws} /(\mathrm{Ws}-\mathrm{Ww})
\end{aligned}
$$

Magnetic susceptibilities were measured using a Geophysica KT-5 susceptibility meter and are reported to $0.01 \times 10-3$ cgs units. The Geophysica KT-5 calculates volume susceptibility by assuming the sample shape is an infinite half-space. The instrument's ability to measure magnetic susceptibility is affected by surface roughness, weathering, and sample size, all of which can result in an underestimation of a sample's true susceptibility. The magnetic susceptibility values reported 
represent an average of multiple (4-8) readings on the sample. Magnetic susceptibility, along with density and rock identification are shown in tables A1a and A1b.

\section{General Discussion}

In general, isostatic gravity anomalies reflect lateral (horizontal) density variations in the middle to upper crust. Thus, gravity anomalies can be used to infer the subsurface structure of known or unknown geologic features. Gravity anomalies often reveal features such as granitic intrusions, calderas, deep sedimentary basins, and faults that may guide groundwater flow or play an important role as aquifers or confining units. Tertiary basaltic rocks are thought to underlie most of the northern and western Smoke Creek Desert region (Dixon and others, 2005), and their subsurface distribution, as highlighted in the magnetic data, is especially important in evaluating the hydrogeology of this area. Cretaceous granitic rocks of the Fox Range, Triassic and Jurassic metasedimentary rocks of the Fox Range, and Quaternary and Tertiary sedimentary deposits that occur throughout the study area also may play a role in the region's hydrology.

In the Smoke Creek Desert area, gravity highs occur over rocks of the Skedaddle Mountains, Fox Range, Granite Range, and over portions of Tertiary volcanic rocks in the Buffalo Hills (fig. 7). These gravity highs likely reflect basement rocks, either exposed at the surface or buried at shallow depths. Average saturated bulk density (SBD) for basement rocks is 2.65, 2.51, and $2.60 \mathrm{~g} / \mathrm{cm}^{3}$ for granitic rocks, andesite, and basalt, respectively (table A1b). The average SBD for metamorphic rocks is $2.66,2.52$, and $2.84 \mathrm{~g} / \mathrm{cm}^{3}$ for gneiss, metasedimentary, and metavolcanic rocks, correspondingly. Gravity lows occur over Smoke Creek Desert, Buffalo and Squaw Creek Valleys, the San Emidio Desert, and to the northwest of the Smoke Creek Desert playa. These lows reflect sedimentary basins filled with lower density alluvial and volcanic deposits. The southern Smoke Creek Desert corresponds to a $25-\mathrm{mGal}$ isostatic gravity low, and assuming a density contrast of $0.4 \mathrm{~g} / \mathrm{cm}^{3}$, an infinite slab approximation yields a basin depth of approximately 2 $\mathrm{km}(1.2 \mathrm{mi})$.

Magnetic anomalies represent lateral variations in the magnetization of rocks. These anomalies can be explained by the variations in rock type across the region. Magnetic highs are likely due to granitic, andesitic, and metavolcanic rocks that have average magnetic susceptibilities of $0.88 \times 10^{-3}, 0.75 \times 10^{-3}$, and $0.77 \times 10^{-3} \mathrm{cgs}$ units, respectively, whereas magnetic lows are probably associated with less magnetic gneiss and metasedimentary rocks (table A1b). Three distinctive patterns of magnetic anomalies occur throughout the Smoke Creek Desert and Squaw Creek Valley (M1-M3, fig. 9). The pattern of magnetic highs and weak magnetic lows in Squaw Creek Valley (fig. 8) likely corresponds to the juxtaposition of magnetic rocks of the Granite Range with weakly-magnetic Tertiary volcanic rocks in the Buffalo Hills along a zone of intense faulting and fracturing in Squaw Creek Valley (M1, fig. 9)). The magnetic anomalies in the northeastern Smoke Creek Desert generally parallel the trend of the Fox Range and the Buffalo Hills (M2, fig. 9). The magnetic highs in the northeastern Smoke Creek Desert likely represent buried granitic rocks. The central and southern Smoke Creek Desert is characterized by a series of east-west trending magnetic anomalies (M3, fig. 9). Magnetic highs in the central and southern Smoke Creek Desert appear to form narrow (2-3 km) E-W trending bands across the desert playa (fig. 9) and probably represent buried volcanic rocks. The northern band correlates with an E-W trending gravity high (fig. 7) and likely represents a buried ridge composed of volcanic or granitic rocks that separates the southern Smoke Creek Desert basin into at least two sub-basins. The southern magnetic band corresponds with the southern limit of a gravity low likely representing the southernmost sub-basin within the Smoke Creek Desert. Areas where the magnetic field is lower include the central Smoke Creek Desert, the southern Smoke Creek Desert, and an area just 
southwest of Gerlach along latitude $40^{\circ} 40^{\prime}$. Contrasting patterns of magnetic anomalies in the Squaw Creek Valley, northeastern Smoke Creek Desert, and the central and southern Smoke Creek Desert reflect the distinctive structural character of those areas.

The diverse physical properties of rock types that outcrop within and likely underlie this region are well suited to geophysical investigations. The contrast in density and magnetic properties between Mesozoic and Paleozoic crystalline basement and overlying Tertiary volcanic rocks and unconsolidated alluvium, for example, produces a distinctive pattern of gravity and magnetic anomalies that can be used to infer geologic structure and determine the depth of preCenozoic basement in three dimensions. Basement topography, which may correspond to the top of buried granitic or metavolcanic rocks, could play an important role in the hydrogeologic framework of the area. 


\section{References Cited}

Barnes, D.F., Oliver, H.W., and Robbins, S.L., 1969, Standardization of gravimeter calibrations in the Geological Survey: Eos, Transactions, American Geophysical Union, v. 50, no. 10, p. 626627.

Blakely, R.J., 1995, Potential Theory in Gravity and Magnetic Applications, Cambridge University Press, New York.

Bonham, H.F., 1969, Geology and mineral deposits of Washoe and Storey Counties, Nevada: Nevada Bureau of Mines and Geology Bulletin 70, 140 p.

Briggs, I.C., 1974, Machine contouring using minimum curvature: Geophysics, v. 39, p. 39-48.

Faulds, J.E., and Ramelli, A.R., in press, Geologic reconnaissance of the Granite Range fault zone and northernmost Smoke Creek Desert, Washoe County, Nevada: Nevada Bureau of Mines and Geology Open-File Report.

Godson, R.H., and Plouff, Donald, 1988, BOUGUER version 1.0, a microcomputer gravityterrain-correction program: U.S. Geological Survey Open-File Report 88-644-A, Documentation, 22 p.; 88-644-B, Tables, 61 p., 88-644-C, 5 1/4 - in diskette.

Hayford, J.F., and Bowie, William, 1912, The effect of topography and isostatic compensation upon the intensity of gravity: U.S. Coast and Geodetic Survey Special Publication no. 10, 132 p.

Hildenbrand, T.G., and Kucks, R.P., 1988, Total intensity magnetic anomaly map of Nevada:

Nevada Bureau of Mines and Geology Map 93A, scale 1:750,000.

International Union of Geodesy and Geophysics, 1971, Geodetic Reference System 1967: International Association of Geodesy Special Publication no. 3, 116 p.

Jachens, R.C., and Roberts, C.W., 1981, Documentation of a FORTRAN program, 'isocomp', for computing isostatic residual gravity: U.S. Geological Open-File Report 81-574, 26 p.

Jennings, C.W., Strand, R.G., and Rogers, T.H., 1977, Geologic map of California: California Division of Mines and Geology, scale 1:750,000.

Langel, R.A., 1992, International geomagnetic reference field: the sixth generation: Journal of Geomagnetism and Geoelectricity, v.44, no. 9, p. 679-707.

Morelli, C., ed, 1974, The International Gravity Standardization Net 1971: International Association of Geodesy Special Publication no. 4, 194 p.

Plouff, Donald, 1966, Digital terrain corrections based on geographic coordinates [abs.]: Geophysics, v. 31, no. 6, p. 1208.

Plouff, Donald, 1977, Preliminary documentation for a FORTRAN program to compute gravity terrain corrections based on topography digitized on a geographic grid: U.S. Geological Survey Open-File Report 77-535, 45 p.

Ponce, D.A., 1997, Gravity data of Nevada: U.S. Geological Survey Digital Data Series DDS-42, 27 p., CD-ROM.

Snyder, D.B., Roberts, C.W., Saltus, R.W., and Sikora, R.F., 1981, Magnetic tape containing the principal facts of 64,402 gravity stations in the State of California: U.S. Geological Survey Report, 30 p.; available from National Technical Information Service, U.S. Department of Commerce, Springfield, Virginia 22161, PB82-168287.

Swick, C.A., 1942, Pendulum gravity measurements and isostatic reductions: U.S. Coast and Geodetic Survey Special Publication 232, 82 p.

Stewart, J.H., and Carlson, J.E., 1978, Geologic map of Nevada: U.S. Geological Survey, scale 1:500,000. 
Stewart, J.H., 1988, Tectonics of the Walker Lane belt, western Great Basin: Mesozoic and Cenozoic deformation in a zone of shear, in Ernst, W.G., ed., Metamorphism and crustal evolution of the western United States: Rubey Volume 7, Prentice-Hall, Inc., Englewood cliffs, N.J., p. 683-713.

Webring, M.W., 1981, MINC-A gridding program based on minimum curvature: U.S. Geological Survey Open File Report 81-1224, 43 p. 


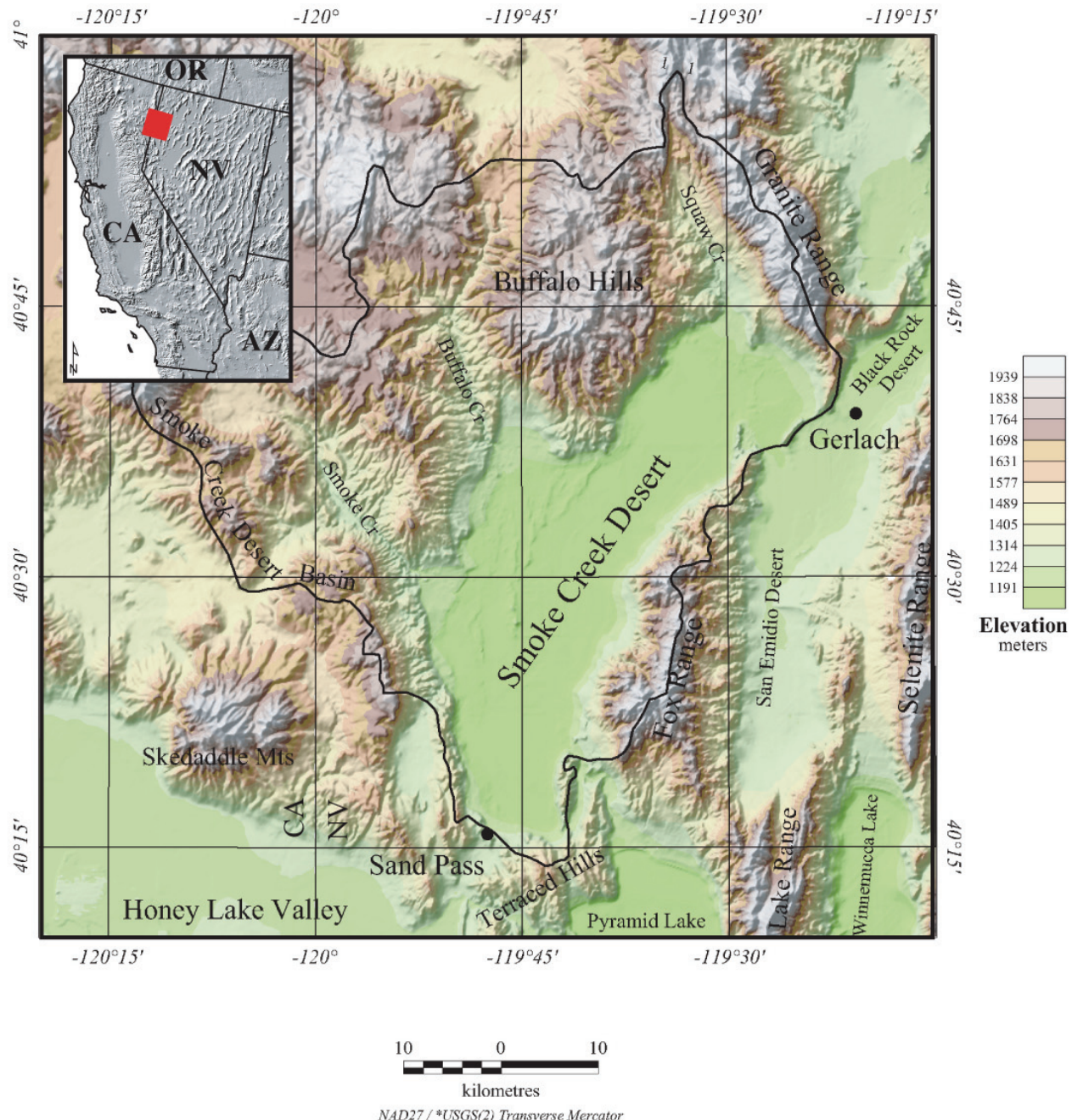

Figure 1. Shaded relief topographic map of the Smoke Creek Basin (outlined in black) and surrounding areas. The index map in the upper left corner shows the study area outlined in red. 

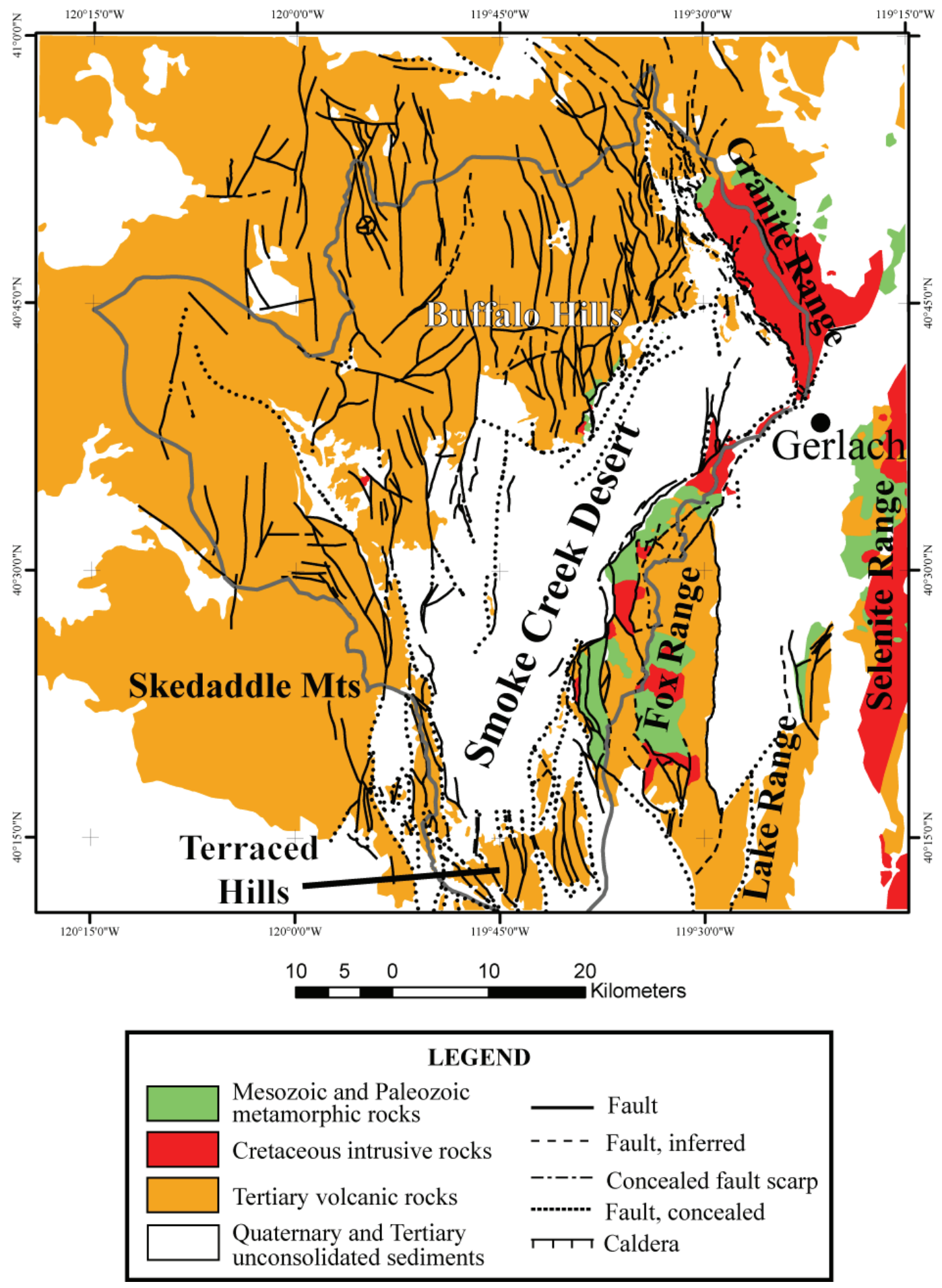

Figure 2. Simplified geologic map of the Smoke Creek Desert area, Nevada (modified after Bonham, 1969; Jennings, 1977; Stewart and Carlson, 1978; G.L. Dixon and others, written commun., 2005; and Faulds and Ramelli, in press) 


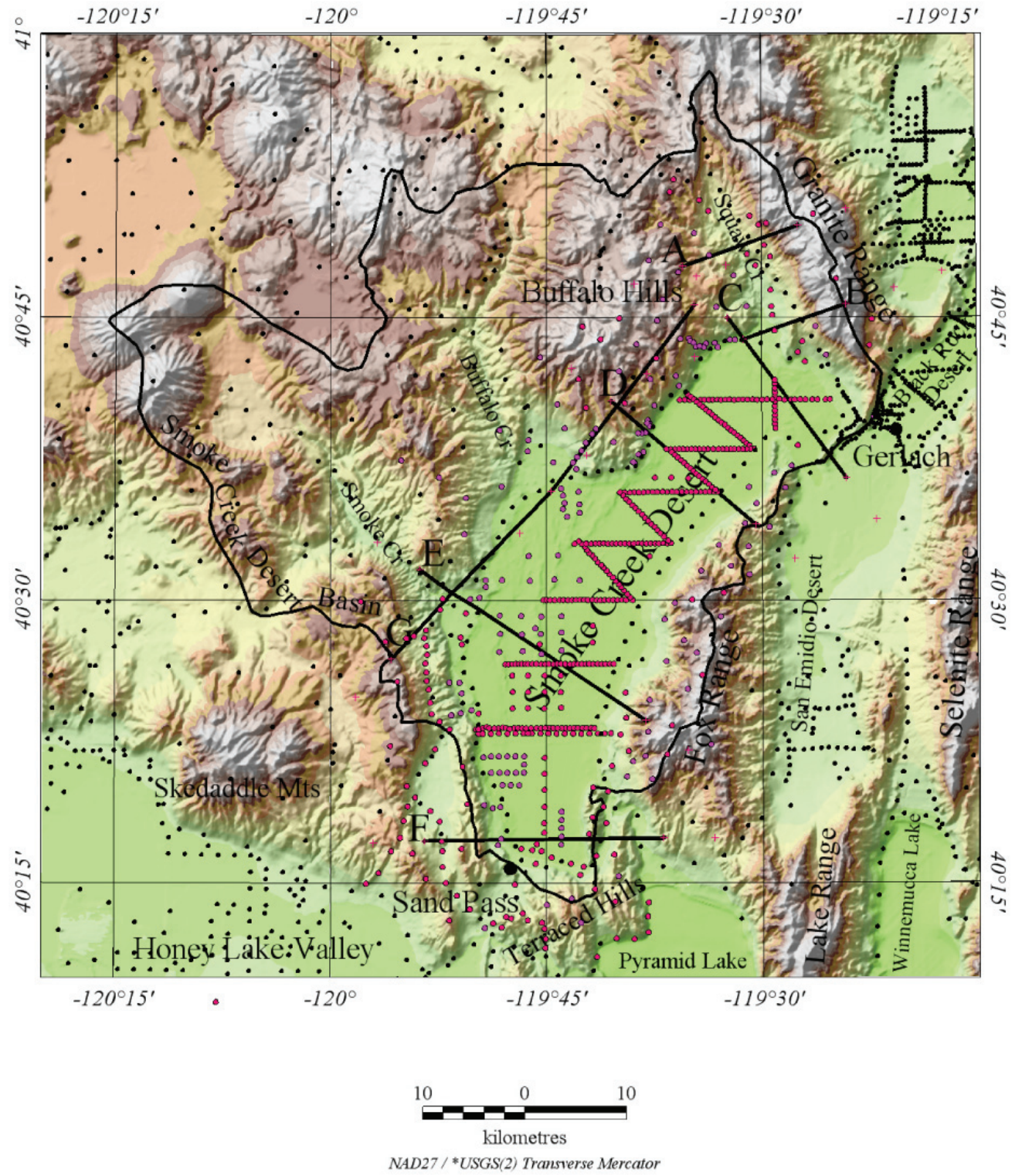

Figure 3. Index map of the Smoke Creek Desert area showing new gravity station locations (magenta), previously collected gravity stations (black), and locations of geologic cross-sections A through $\mathrm{G}$. 


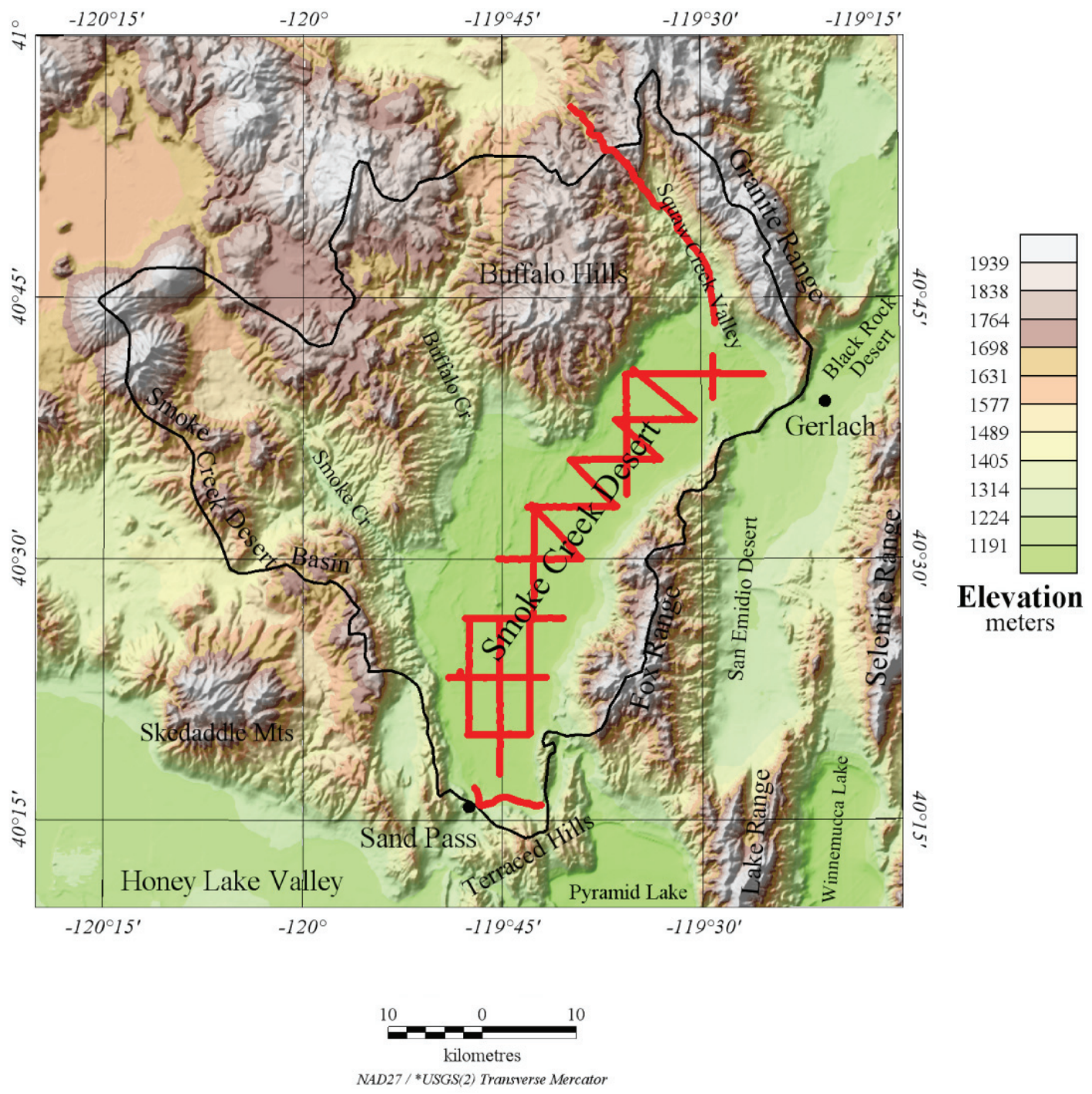

Figure 4. Topographic map showing the truck-towed magnetometer transects (red) within the Smoke Creek Desert and Squaw Creek Valley. 

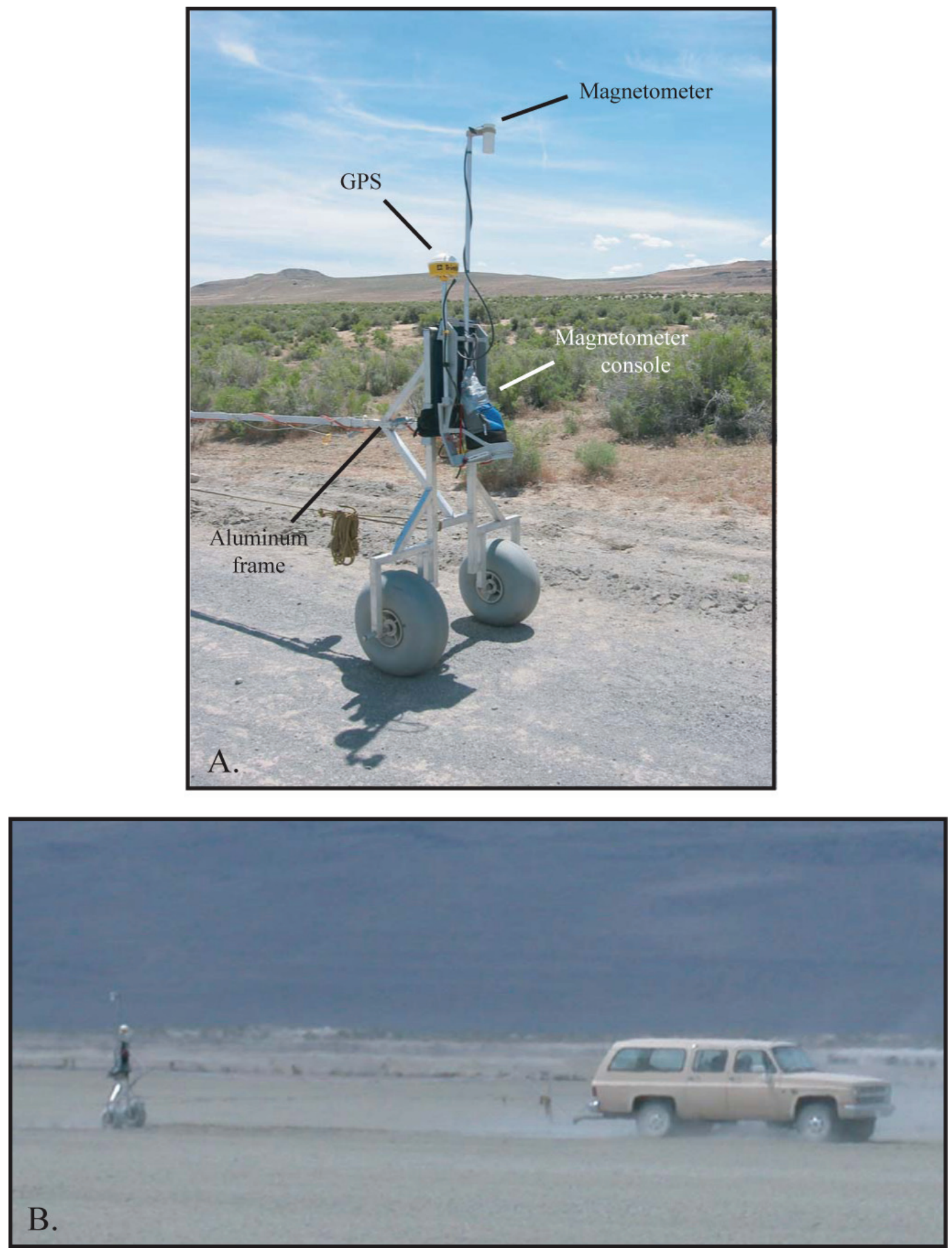

Figure 5. Truck-towed magnetometer system used in this study. The G-858 cesium magnetometer and GPS unit (A) being towed along a transect in the Smoke Creek Desert (B). 


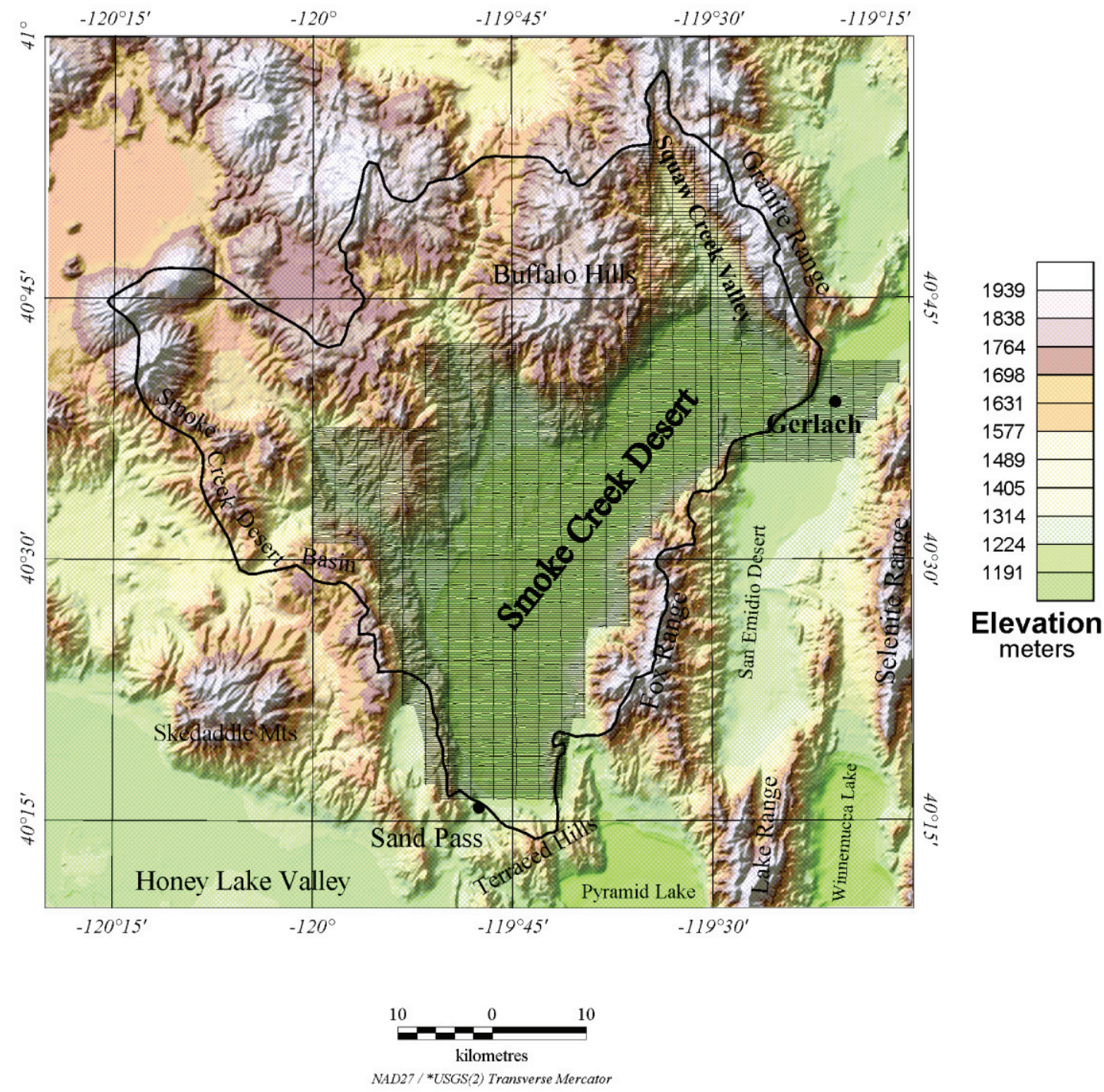

Figure 6. Topographic map showing the flight lines (black) for the detailed aeromagnetic survey in the Smoke Creek Desert and Squaw Creek Valley. 


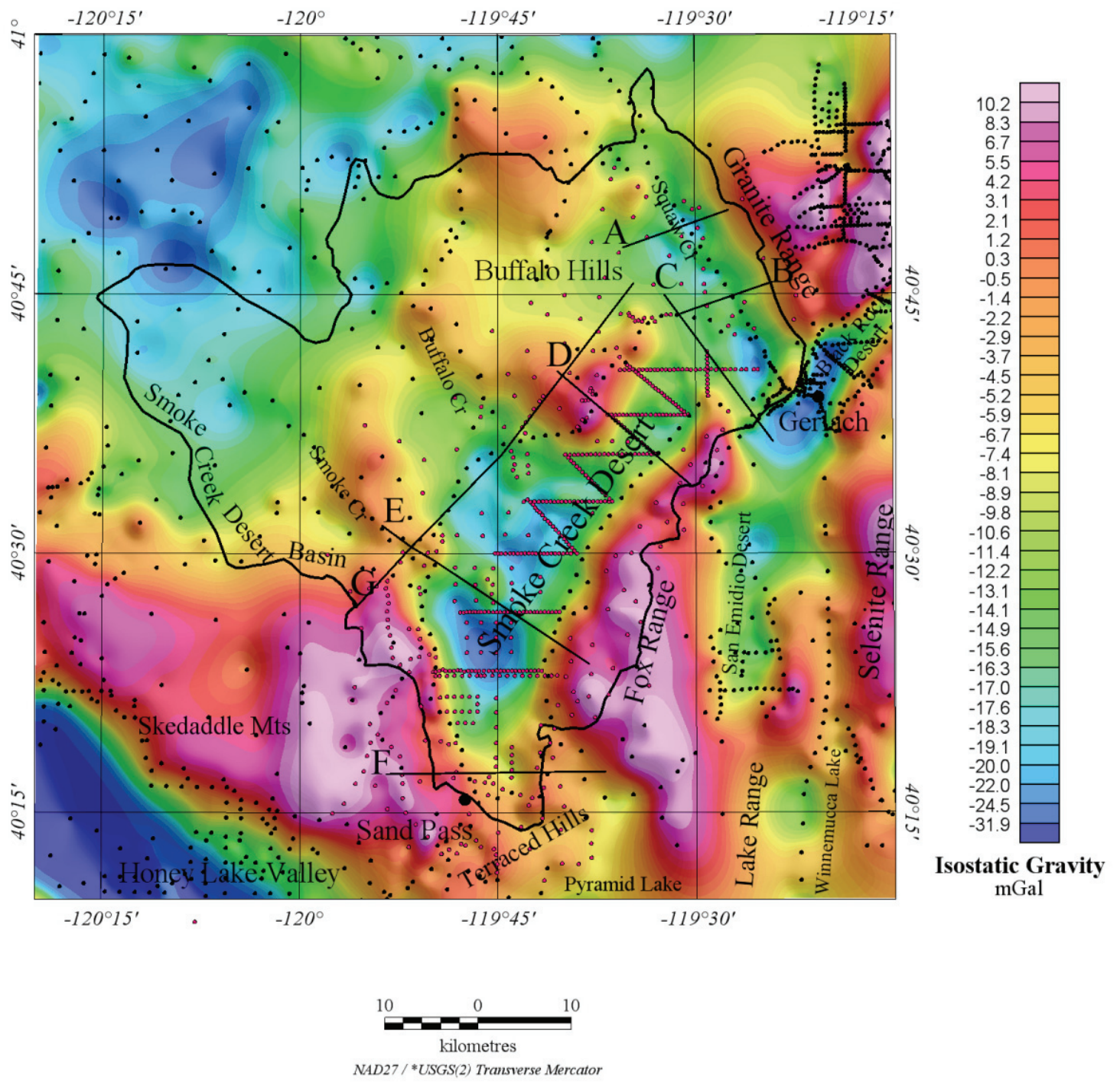

Figure 7. Isostatic gravity map of the Smoke Creek Desert area showing new gravity station locations (magenta) and previously collected gravity stations (black). 


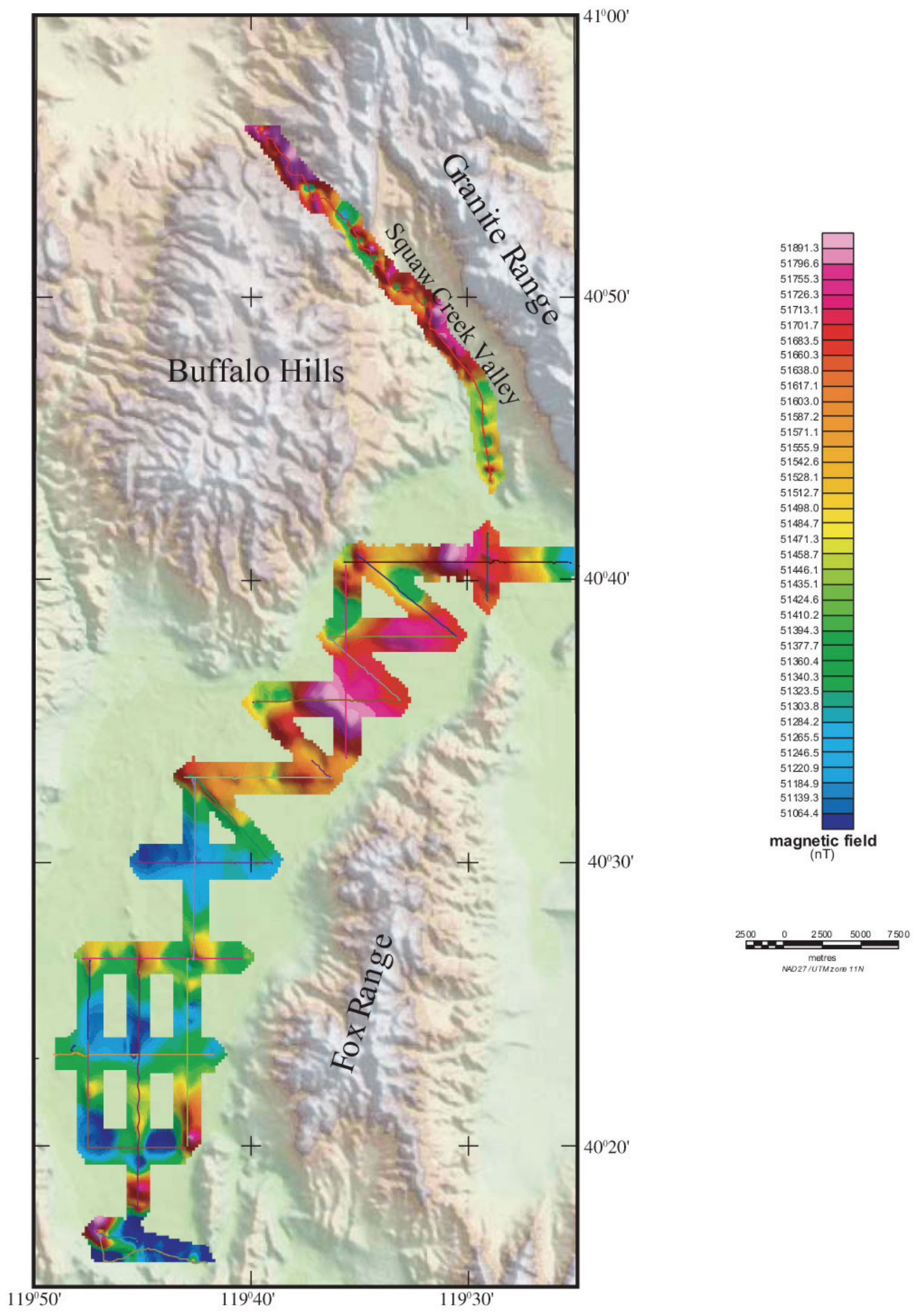

Figure 8. Contour map of towed-magnetometer data from the Smoke Creek Desert and Squaw Creek Valley. 


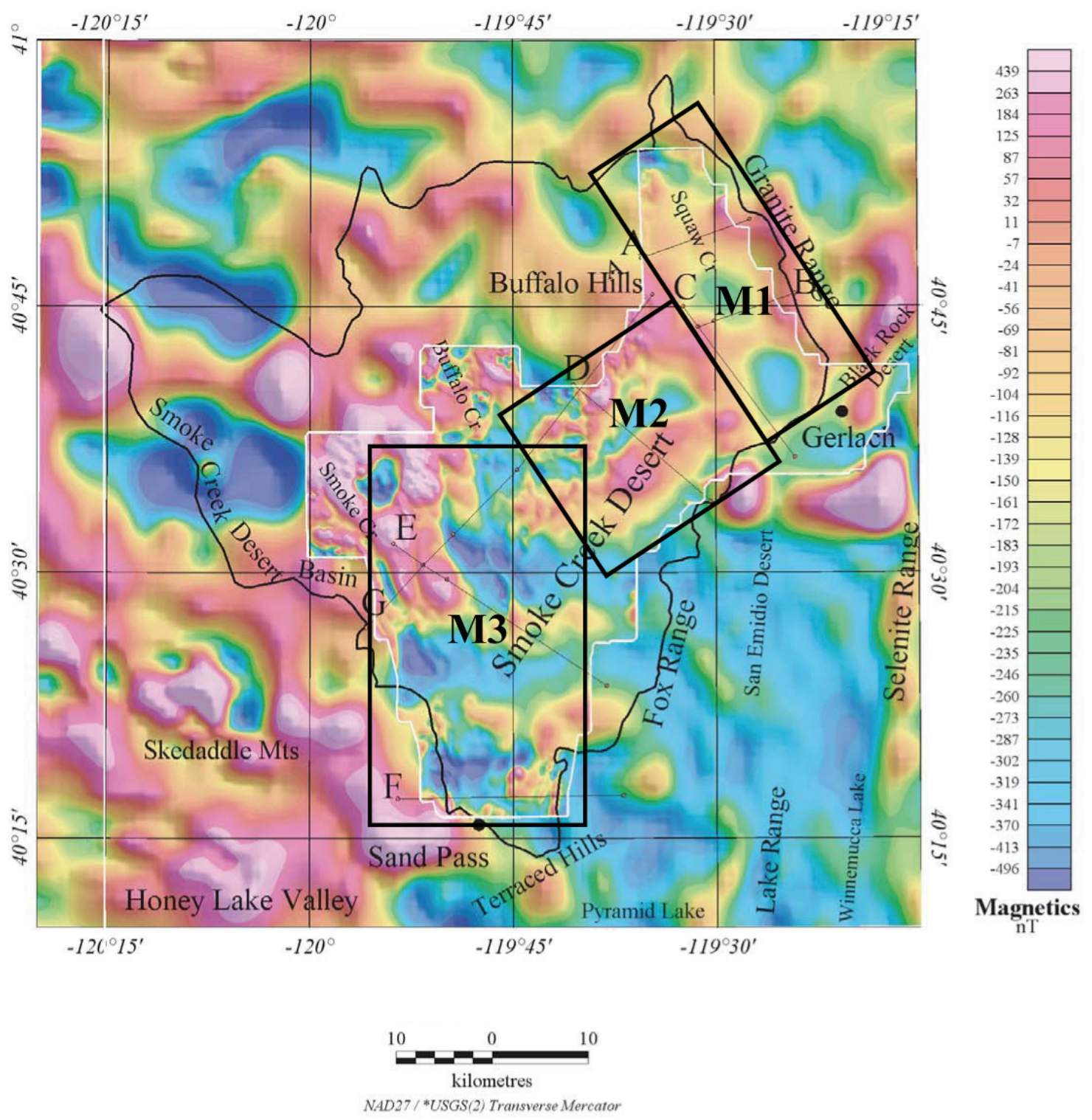

Figure 9. Aeromagnetic map of the Smoke Creek Desert area showing the detailed aeromagnetic survey (outlined in white) superimposed on the regional aeromagnetic map of Nevada and California (Hildenbrand and Kucks, 1988). 


\section{Appendix}

Table A1a. Physical property measurements of rock samples in the Smoke Creek Desert area. [Latitudes (north) and longitudes (west) are on the North American Datum 1927 (NAD27). DBD, dry bulk density; GD, grain density; SBD, saturated bulk density. All samples correspond with gravity station locations. Precision for location, density, and susceptibility is 0.01 .]

\begin{tabular}{|c|c|c|c|c|c|c|}
\hline \multirow[t]{2}{*}{ Station ID } & \multirow{2}{*}{$\begin{array}{l}\text { Latitude } \\
\text { deg min }\end{array}$} & \multirow{2}{*}{$\begin{array}{l}\text { Longitude } \\
\text { deg min }\end{array}$} & \multicolumn{3}{|c|}{ Density $\left(\mathrm{g} / \mathrm{cm}^{3}\right)$} & \multirow{2}{*}{$\begin{array}{l}\text { Susceptibility } \\
\left(10^{-3} \text { cgs units }\right)\end{array}$} \\
\hline & & & GD & SBD & DBD & \\
\hline \multicolumn{7}{|c|}{ Tertiary Andesite } \\
\hline 04LOV018 & 4015.01 & 11951.99 & 2.61 & 2.47 & 2.38 & 1.05 \\
\hline 04LOV025 & 4020.14 & 11954.69 & 2.63 & 2.53 & 2.47 & 0.90 \\
\hline 04LOV031 & 4024.57 & 11953.07 & 2.77 & 2.74 & 2.73 & 1.46 \\
\hline 04LOV221 & 4025.10 & 11939.51 & 2.69 & 2.66 & 2.64 & 0.64 \\
\hline 04LOV320 & 4040.21 & 11942.21 & 2.65 & 2.59 & 2.55 & 0.48 \\
\hline 04LOV325 & 4042.83 & 11937.31 & 2.80 & 2.74 & 2.71 & 1.21 \\
\hline 04LOV337 & 4021.17 & 11950.82 & 2.56 & 2.50 & 2.46 & 0.81 \\
\hline 04LOV506 & 4030.40 & 11932.66 & 2.37 & 2.29 & 2.22 & 0.32 \\
\hline 05DAP101 & 4043.67 & 11933.88 & 2.57 & 2.48 & 2.43 & 1.24 \\
\hline 05LOV002 & 4012.89 & 11942.62 & 2.30 & 2.11 & 1.97 & 1.20 \\
\hline 05LOV017 & 4032.85 & 11930.93 & 2.49 & 2.47 & 2.46 & 0.40 \\
\hline \multicolumn{7}{|c|}{ Tertiary Basalt } \\
\hline 04LOV033 & 4025.73 & 11953.31 & 2.88 & 2.85 & 2.84 & 0.82 \\
\hline 04LOV035 & 4018.29 & 11954.90 & 2.62 & 2.52 & 2.46 & 0.38 \\
\hline 04LOV218 & 4022.00 & 11939.29 & 2.73 & 2.71 & 2.69 & 0.56 \\
\hline $04 \mathrm{LOV} 223$ & 4017.60 & 11942.05 & 2.71 & 2.69 & 2.68 & 0.09 \\
\hline 04LOV234 & 4041.67 & 11929.06 & 2.52 & 2.41 & 2.33 & 0.55 \\
\hline $04 \mathrm{LOV} 315$ & 4027.58 & 11953.32 & 2.73 & 2.64 & 2.59 & 0.93 \\
\hline 04LOV 319 & 4027.67 & 11945.47 & 2.25 & 2.20 & 2.17 & 0.36 \\
\hline 04LOV321 & 4041.73 & 11942.74 & 1.62 & 1.50 & 1.32 & 0.04 \\
\hline 04LOV322 & 4044.52 & 11942.45 & 2.45 & 2.38 & 2.34 & 0.55 \\
\hline 04LOV324 & 4043.85 & 11941.19 & 2.77 & 2.67 & 2.61 & 0.65 \\
\hline 04LOV336 & 4022.27 & 11955.84 & 2.72 & 2.66 & 2.62 & 0.54 \\
\hline 04LOV338 & 4019.19 & 11950.50 & 2.58 & 2.52 & 2.47 & 0.66 \\
\hline 04LOV339 & 4016.83 & 11950.90 & 2.54 & 2.48 & 2.44 & 0.05 \\
\hline 04LOV340 & 4016.57 & 11954.31 & 2.79 & 2.74 & 2.70 & 0.51 \\
\hline 04LOV341 & 4015.22 & 11953.36 & 2.36 & 2.32 & 2.30 & 0.21 \\
\hline 04LOV342 & 4013.02 & 11951.75 & 2.26 & 2.08 & 1.95 & 0.23 \\
\hline $04 \mathrm{LOV} 413$ & 4052.32 & 11936.08 & 2.53 & 2.41 & 2.34 & 0.68 \\
\hline 04LOV414 & 4051.66 & 11936.34 & 2.77 & 2.75 & 2.74 & 0.40 \\
\hline
\end{tabular}




\begin{tabular}{|c|c|c|c|c|c|c|}
\hline 04LOV415 & 4051.22 & 11934.28 & 2.64 & 2.61 & 2.60 & 0.56 \\
\hline 04LOV500 & 4014.67 & 11941.64 & 2.56 & 2.52 & 2.49 & 0.53 \\
\hline 04LOV501 & 4016.51 & 11940.31 & 2.68 & 2.63 & 2.60 & 0.42 \\
\hline 04LOV502 & 4018.48 & 11941.81 & 2.76 & 2.74 & 2.73 & 0.09 \\
\hline 04LOV503 & 4021.82 & 11936.61 & 2.76 & 2.70 & 2.67 & 0.66 \\
\hline 04LOV504 & 4023.36 & 11936.28 & 2.36 & 2.29 & 2.24 & 0.91 \\
\hline 04LOV505 & 4026.03 & 11934.18 & 2.72 & 2.71 & 2.70 & 0.01 \\
\hline 04LOV507 & 4030.03 & 11934.86 & 2.74 & 2.71 & 2.69 & 0.02 \\
\hline 05LOV001 & 4013.84 & 11944.99 & 2.77 & 2.74 & 2.72 & 0.65 \\
\hline 05LOV003 & 4014.17 & 11943.27 & 2.67 & 2.65 & 2.63 & 0.51 \\
\hline 05LOV006 & 4023.02 & 11937.94 & 2.47 & 2.40 & 2.36 & 0.66 \\
\hline 05LOV007 & 4022.34 & 11933.56 & 2.86 & 2.85 & 2.85 & 0.59 \\
\hline 05LOV008 & 4025.29 & 11935.18 & 2.63 & 2.61 & 2.61 & 0.71 \\
\hline 05LOV009B & 4024.71 & 11937.84 & 2.37 & 2.31 & 2.26 & 0.42 \\
\hline 05LOV011 & 4027.21 & 11934.55 & 2.80 & 2.79 & 2.78 & 1.70 \\
\hline 05LOV028 & 4049.47 & 11934.65 & 2.68 & 2.65 & 2.63 & 0.26 \\
\hline 05LOV030 & 4048.02 & 11937.81 & 2.75 & 2.69 & 2.65 & 1.16 \\
\hline 05LOV031 & 4047.40 & 11935.76 & 2.25 & 2.18 & 2.12 & 0.30 \\
\hline 05LOV032 & 4048.30 & 11933.61 & 2.75 & 2.71 & 2.69 & 0.41 \\
\hline 05LOV033 & 4047.22 & 11931.90 & 2.37 & 2.30 & 2.25 & 0.52 \\
\hline 05LOV034 & 4045.59 & 11931.02 & 2.75 & 2.72 & 2.71 & 0.48 \\
\hline 05LOV035 & 4045.55 & 11933.12 & 2.77 & 2.74 & 2.72 & 1.23 \\
\hline 05LOV037 & 4044.84 & 11937.25 & 2.64 & 2.56 & 2.52 & 0.84 \\
\hline 05LOV038 & 4043.86 & 11935.63 & 1.85 & 1.82 & 1.77 & 0.19 \\
\hline 05LOV039 & 4043.58 & 11938.69 & 2.84 & 2.81 & 2.80 & 0.98 \\
\hline 05LOV040 & 4041.73 & 11937.73 & 2.62 & 2.56 & 2.52 & 1.58 \\
\hline 05LOV041 & 4042.15 & 11939.92 & 2.61 & 2.58 & 2.55 & 0.69 \\
\hline 05LOV042 & 4042.89 & 11945.41 & 2.68 & 2.62 & 2.58 & 1.11 \\
\hline 05LOV047 & 4037.43 & 11955.08 & 2.72 & 2.70 & 2.68 & 1.24 \\
\hline 05LOV048 & 4036.51 & 11952.45 & 2.65 & 2.61 & 2.59 & 1.01 \\
\hline 05LOV070A & 4020.65 & 11939.41 & 2.87 & 2.85 & 2.85 & 0.10 \\
\hline 05LOV070B & 4020.65 & 11939.41 & 2.86 & 2.86 & 2.85 & 0.12 \\
\hline 05LOV070C & 4020.65 & 11939.41 & 2.88 & 2.88 & 2.87 & 0.52 \\
\hline 05LOV5A & 4043.41 & 11933.01 & 2.81 & 2.80 & 2.80 & 0.58 \\
\hline 05LOV5B & 4043.41 & 11933.01 & 2.80 & 2.79 & 2.79 & 0.54 \\
\hline 05LOV5C & 4043.41 & 11933.01 & 2.84 & 2.83 & 2.83 & 0.49 \\
\hline 05SCD002 & 4027.67 & 11945.47 & 2.89 & 2.89 & 2.88 & 0.12 \\
\hline 05SCD003 & 4027.67 & 11945.47 & 2.90 & 2.89 & 2.88 & 0.12 \\
\hline 05SCD004 & 4027.67 & 11945.47 & 2.89 & 2.89 & 2.88 & 0.12 \\
\hline
\end{tabular}




\begin{tabular}{|c|c|c|c|c|c|c|}
\hline \multicolumn{7}{|c|}{ Tertiary Conglomerate } \\
\hline 05SCD001 & 4027.67 & 11945.47 & 2.78 & 2.77 & 2.77 & 0.02 \\
\hline \multicolumn{7}{|c|}{ Cretaceous Diorite } \\
\hline 05LOV019 & 4035.13 & 11930.36 & 2.78 & 2.73 & 2.70 & 1.23 \\
\hline 05LOV021B & 4036.75 & 11929.13 & 2.87 & 2.84 & 2.82 & 0.15 \\
\hline 05LOV022 & 4037.12 & 11927.91 & 2.59 & 2.55 & 2.52 & 0.03 \\
\hline 05LOV023B & 4035.96 & 11928.35 & 2.78 & 2.71 & 2.68 & 0.73 \\
\hline 05LOV075 & 4041.90 & 11935.95 & 2.70 & 2.67 & 2.65 & 0.70 \\
\hline 05LOV5D & 4043.41 & 11933.01 & 2.81 & 2.80 & 2.80 & 0.42 \\
\hline \multicolumn{7}{|c|}{ Mesozoic and Paleozoic Gneiss } \\
\hline 05LOV018 & 4034.07 & 11931.16 & 2.70 & 2.62 & 2.57 & 0.01 \\
\hline 05LOV024 & 4033.88 & 11929.46 & 2.74 & 2.70 & 2.67 & 0.01 \\
\hline \multicolumn{7}{|c|}{ Cretaceous Granitic } \\
\hline 04LOV224 & 4050.48 & 11926.34 & 2.69 & 2.67 & 2.66 & 0.09 \\
\hline 04LOV225 & 4047.04 & 11924.75 & 2.69 & 2.65 & 2.62 & 1.01 \\
\hline 04LOV226 & 4044.89 & 11922.28 & 2.79 & 2.72 & 2.69 & 0.88 \\
\hline 04LOV227 & 4043.51 & 11922.46 & 2.70 & 2.67 & 2.66 & 0.68 \\
\hline 04LOV407 & 4047.75 & 11930.38 & 2.67 & 2.64 & 2.62 & 1.01 \\
\hline 04LOV501 & 4016.51 & 11940.31 & 2.68 & 2.63 & 2.60 & 0.42 \\
\hline 05LOV503A & 4037.74 & 11938.57 & 2.69 & 2.65 & 2.63 & 3.75 \\
\hline 05LOV521A & 4037.33 & 11938.94 & 2.68 & 2.63 & 2.61 & 0.72 \\
\hline 05LOV521B & 4037.33 & 11938.94 & 2.70 & 2.67 & 2.65 & 0.64 \\
\hline 05LOV015 & 4031.56 & 11934.23 & 2.60 & 2.60 & 2.59 & 0.00 \\
\hline 05LOV021A & 4036.75 & 11929.13 & 2.63 & 2.61 & 2.60 & 0.00 \\
\hline 05LOV010 & 4026.76 & 11936.66 & 2.74 & 2.70 & 2.67 & 0.52 \\
\hline 05LOV016A & 4032.93 & 11932.99 & 2.61 & 2.60 & 2.59 & 0.00 \\
\hline \multicolumn{7}{|c|}{ Mesozoic and Paleozoic Marble } \\
\hline 05LOV072 & 4021.73 & 11938.13 & 2.68 & 2.67 & 2.66 & 0.00 \\
\hline \multicolumn{7}{|c|}{ Mesozoic and Paleozoic Metasedimentary } \\
\hline 05LOV005 & 4020.52 & 11937.66 & 2.65 & 2.62 & 2.60 & 0.01 \\
\hline 05LOV9A1 & 4024.71 & 11937.84 & 2.35 & 1.96 & 1.67 & 0.06 \\
\hline 05LOV9A2 & 4024.71 & 11937.84 & 2.36 & 1.98 & 1.69 & 0.12 \\
\hline 05LOV014 & 4029.86 & 11936.06 & 2.76 & 2.74 & 2.73 & 0.02 \\
\hline 05LOV016B & 4032.93 & 11932.99 & 2.56 & 2.50 & 2.46 & 0.00 \\
\hline 05LOV023A & 4035.96 & 11928.35 & 2.83 & 2.82 & 2.82 & 0.02 \\
\hline 05LOV076 & 4040.32 & 11937.45 & 3.05 & 3.04 & 3.03 & 0.60 \\
\hline \multicolumn{7}{|c|}{ Mezozoic and Paleozoic Metavolcanic } \\
\hline 05LOV500 & 4039.39 & 11937.81 & 2.76 & 2.75 & 2.75 & 1.42 \\
\hline 05LOV500B & 4039.39 & 11937.81 & 2.84 & 2.83 & 2.82 & 1.81 \\
\hline
\end{tabular}




\begin{tabular}{lllllll} 
05LOV502A & 4037.86 & 11938.22 & 2.95 & 2.94 & 2.93 & 2.07 \\
05LOV502B & 4037.86 & 11938.22 & 2.95 & 2.95 & 2.94 & 1.77 \\
05LOV503B & 4037.74 & 11938.57 & 2.78 & 2.75 & 2.74 & 0.02 \\
05LOV504A & 4043.41 & 11933.01 & 2.81 & 2.76 & 2.74 & 0.38 \\
05LOV516A & 4039.40 & 11937.96 & 2.86 & 2.85 & 2.84 & 0.02 \\
05LOV516B & 4039.40 & 11937.96 & 2.81 & 2.80 & 2.80 & 0.03 \\
05LOV519 & 4038.86 & 11938.33 & 3.03 & 3.02 & 3.02 & 0.03 \\
05DAP102 & 4039.47 & 11937.93 & 2.69 & 2.67 & 2.66 & 0.92 \\
05DAP103 & 4038.42 & 11938.42 & 2.96 & 2.95 & 2.94 & 0.04 \\
\hline
\end{tabular}

Table A1b. Mean physical property data by rock type.

\begin{tabular}{|c|c|c|c|c|c|}
\hline \multirow[b]{2}{*}{ Rock Type } & \multirow{2}{*}{$\begin{array}{l}\text { No. of } \\
\text { samples }\end{array}$} & \multicolumn{3}{|c|}{ Density $\left(\mathrm{g} / \mathrm{cm}^{3}\right)$} & \multirow{2}{*}{$\begin{array}{c}\text { Susceptibility }\left(10^{-3} \mathrm{cgs}\right. \\
\text { units })\end{array}$} \\
\hline & & Grain & $\begin{array}{l}\text { Saturated } \\
\text { bulk }\end{array}$ & $\begin{array}{l}\text { Dry } \\
\text { bulk }\end{array}$ & \\
\hline Andesite & 11 & 2.59 & 2.51 & 2.46 & 0.88 \\
\hline Basalt & 56 & 2.64 & 2.59 & 2.56 & 0.55 \\
\hline Diorite & 6 & 2.76 & 2.72 & 2.70 & 0.54 \\
\hline Granitic & 13 & 2.68 & 2.65 & 2.63 & 0.75 \\
\hline \multicolumn{6}{|l|}{ Metamorphic } \\
\hline Gneiss & 2 & 2.72 & 2.66 & 2.62 & 0.01 \\
\hline Metasedimentary & 7 & 2.65 & 2.52 & 2.43 & 0.12 \\
\hline Metavolcanic & 11 & 2.86 & 2.84 & 2.83 & 0.77 \\
\hline
\end{tabular}


Table A2. Principal facts of gravity stations in the Smoke Creek Desert area. [Latitudes (north) and longitudes (west) are on the North American Datum 1927 (NAD27). Elevations are on the North AmericanVertical Datum 1929 (NAVD29). CBA, complete Bouguer anomaly; FAA, free-air anomaly; ISO, isostatic anomaly; ITC, inner terrain correction; OG, observed gravity; SBA, simple Bouguer anomaly; TTC, total terrain correction.]

\begin{tabular}{|c|c|c|c|c|c|c|c|c|c|c|c|c|}
\hline \multirow[t]{2}{*}{ Station } & \multicolumn{2}{|c|}{ Latitude } & \multicolumn{2}{|c|}{ Longitude } & \multirow{2}{*}{$\begin{array}{c}\text { Elevation } \\
\mathrm{ft}\end{array}$} & \multirow{2}{*}{$\begin{array}{c}\mathrm{OG} \\
\mathrm{mGal}\end{array}$} & \multirow{2}{*}{$\begin{array}{l}\text { FAA } \\
\text { mGal }\end{array}$} & \multirow{2}{*}{$\begin{array}{l}\text { SBA } \\
\mathrm{mGal}\end{array}$} & \multirow{2}{*}{$\begin{array}{c}\text { ITC } \\
\mathrm{mGal}\end{array}$} & \multirow{2}{*}{$\begin{array}{l}\text { TTC } \\
\mathrm{mGal} \\
\end{array}$} & \multirow{2}{*}{$\begin{array}{l}\mathrm{CBA} \\
\mathrm{mGal} \\
\end{array}$} & \multirow{2}{*}{$\begin{array}{l}\text { ISO } \\
\mathrm{mGal}\end{array}$} \\
\hline & deg & $\min$ & deg & $\min$ & & & & & & & & \\
\hline 04LOV001 & 40 & 12.62 & 119 & 49.27 & 4043 & 979796.0 & -11.6 & -149.5 & 0.0 & 0.5 & -150.3 & 1.0 \\
\hline 04LOV002 & 40 & 2.93 & 119 & & & & -21.0 & & 0.2 & & & -1.7 \\
\hline 04LOV003 & 40 & 22.92 & 119 & 49.42 & 47 & 979814.2 & -27.2 & -158.3 & 0.0 & 0.9 & -158.7 & -7.0 \\
\hline 04LOV004 & 40 & 22.93 & 119 & & & 979809.9 & & -161.3 & 0.0 & 0.4 & -162.1 & -10.6 \\
\hline 04LOV005 & 40 & 22.94 & 119 & & & 979808.6 & 1.0 & -162.9 & 0.0 & 0.3 & -163.8 & -12.3 \\
\hline 04LOV006 & 40 & 22.93 & 119 & 47.71 & 50 & 979805.5 & -35.5 & -166.8 & 0.0 & 0.2 & -167.8 & -16.3 \\
\hline 04LOV007 & 40 & 22.94 & 119 & 47.15 & 3844 & 979801.5 & -40.0 & -171.2 & 0.0 & 0.2 & -172.2 & -20.7 \\
\hline $04 \mathrm{LO}$ & 40 & 2.94 & 119 & & 345 & & & & 0.0 & .2 & -174.2 & -22.8 \\
\hline $04 \mathrm{LO}$ & 40 & 22.94 & 119 & & & & & & 0.0 & .2 & -175.0 & -23.6 \\
\hline 04LOV010 & 40 & 22.94 & 119 & 45. & & 979 & -42.7 & & 0.0 & 0.2 & -174.9 & -23.5 \\
\hline 04LOV011 & 40 & 22.94 & 119 & 44.8 & & 97980 & -41.5 & -172.6 & 0.0 & 0.2 & -173.7 & -22.3 \\
\hline 04LOV012 & 40 & 22.93 & 119 & 44.3 & & 97980 & -40.1 & -171.4 & 0.0 & 0.2 & -172.4 & -21.1 \\
\hline 04LOV013 & 40 & 22.93 & 119 & 43.7 & 51 & 97980 & -39.2 & & 0.0 & .3 & -171.5 & -20.1 \\
\hline & 40 & & 119 & & & & & & & 4 & & -18.4 \\
\hline & 40 & 22. & 119 & & & & & & & 2 & & -23.9 \\
\hline & 40 & 13.61 & 119 & & & & 7.3 & & & 0.6 & & 14.4 \\
\hline $04 \mathrm{LO}$ & 40 & 14.33 & 119 & 51. & & 9797 & 0.3 & & & 0.8 & -145.2 & 6.0 \\
\hline $04 \mathrm{LO}$ & 40 & & 119 & & & & 9.1 & -142 & & 9 & -142 & 8.4 \\
\hline & 40 & & 119 & & & & & & & & -14 & 9.9 \\
\hline & 40 & & 119 & & & & & & & 9 & & 9.9 \\
\hline & 40 & 17.21 & 119 & & & & 4.5 & & & .7 & & 8.4 \\
\hline 04LO & 40 & & 119 & & & & & & & .5 & & 7.5 \\
\hline $04 \mathrm{LO}$ & 40 & 18.8 & 119 & & & & & & & .6 & -14 & 7.2 \\
\hline & 40 & 19.5 & 119 & & & & & & & & -143 & 7.8 \\
\hline & 40 & 2 & 119 & & & & 10.6 & & & 7 & & 10.4 \\
\hline $04 \mathrm{LO}$ & 40 & 21.36 & 119 & & & & & & & 7 & & 7.7 \\
\hline & 40 & 22.10 & 119 & & & & -0.6 & & 0.1 & 1.5 & -144.2 & 7.4 \\
\hline & 40 & 23.15 & 119 & & & & & & & 1.0 & -142.1 & 9.6 \\
\hline & 40 & & 119 & & & & & & & 5 & -139 & 11.9 \\
\hline & 40 & 23.9 & 119 & & & & 12.9 & & 0 . & .0 & -13 & 12.9 \\
\hline 04LO & 40 & 24.5 & 119 & & & & 10.5 & & & .3 & -139.2 & 12.9 \\
\hline & 40 & & 119 & & & & 7.5 & & 0.4 & 2.5 & -141.6 & 10.6 \\
\hline 04LOV033 & 40 & 25.73 & 119 & & & & 10.6 & -144.6 & 0.8 & 2.6 & -143.4 & 8.8 \\
\hline & 40 & 19.06 & 119 & & & & & & & & & 10.2 \\
\hline & 40 & 18.29 & 11 & & & & & & & .5 & -140 & 10.5 \\
\hline & 40 & 17.40 & 119 & 55.14 & 4850 & 979763.6 & 24.8 & -140.6 & 0.8 & 1.8 & -140.3 & 10.8 \\
\hline 04LOV037 & 40 & 16.69 & 119 & 55.78 & & & 18.8 & & 2.0 & 3.2 & & 11.8 \\
\hline 04LOV038 & 40 & 15.53 & 119 & 56.64 & & 979794.2 & 8.0 & -139.2 & 0.1 & 1.0 & -139.5 & 11.8 \\
\hline 04LOV039 & 40 & 14.97 & 119 & & 4142 & 979802.0 & 0.3 & -141 & 0.1 & 0.7 & -141.6 & 9.8 \\
\hline & 40 & 22.93 & 119 & & & & & & 0.0 & 0.5 & -165.9 & -14.7 \\
\hline & 40 & 22.93 & 119 & 42.02 & 3872 & 979812.5 & -26.5 & & 0.0 & 0.7 & -159.1 & -7.8 \\
\hline 04LOV042 & 40 & 22.93 & 119 & 41.44 & 3891 & 979814.9 & -22.2 & -155.0 & 0.0 & 1.0 & -155.2 & -4.0 \\
\hline
\end{tabular}




\begin{tabular}{|c|c|c|c|c|c|c|c|c|c|c|c|c|}
\hline 04LOV043 & 40 & 22.95 & 119 & 40.53 & 4034 & 979809.5 & -14.3 & -151.9 & 0.6 & 2.5 & -150.7 & 0.6 \\
\hline 04LOV044 & 40 & 22.93 & 119 & 40.96 & 3939 & 979814.9 & -17.7 & -152.0 & 0.0 & 1.4 & -151.9 & -0.7 \\
\hline 04LOV045 & 40 & 19.86 & 119 & 40.73 & 3928 & 979809.2 & -20.0 & -153.9 & 0.1 & 1.2 & -153.9 & -2.9 \\
\hline 04LOV046 & 40 & 19.04 & 119 & 41.05 & 3937 & 979809.1 & -18.0 & -152.2 & 0.3 & 1.4 & -152.1 & -1.1 \\
\hline 04LOV047 & 40 & 18.18 & 119 & 40.86 & 3975 & 979806.2 & -16.1 & -151.7 & 0.0 & 1.0 & -151.9 & -1.0 \\
\hline 04LOV048 & 40 & 16.44 & 119 & 41.56 & 3956 & 979806.7 & -14.7 & -149.6 & 0.1 & 0.9 & -150.0 & 0.8 \\
\hline 04LOV049 & 40 & 15.86 & 119 & 42.43 & 3957 & 979805.4 & -15.1 & -150.0 & 0.0 & 0.8 & -150.5 & 0.4 \\
\hline 04LOV050 & 40 & 15.43 & 119 & 42.68 & 4015 & 979801.6 & -12.8 & -149.7 & 0.1 & 1.4 & -149.6 & 1.3 \\
\hline 04LOV051 & 40 & 16.03 & 119 & 43.32 & 3894 & 979805.4 & -21.2 & -154.0 & 0.0 & 0.8 & -154.5 & -3.6 \\
\hline 04LOV052 & 40 & 16.35 & 119 & 44.66 & 3878 & 979806.0 & -22.6 & -154.9 & 0.0 & 0.5 & -155.6 & -4.7 \\
\hline 04LOV053 & 40 & 16.02 & 119 & 45.76 & 3976 & 979800.9 & -18.1 & -153.7 & 0.0 & 0.4 & -154.5 & -3.6 \\
\hline 04LOV054 & 40 & 14.92 & 119 & 47.20 & 4121 & 979796.0 & -7.6 & -148.1 & 0.0 & 0.4 & -149.1 & 2.0 \\
\hline 04LOV055 & 40 & 12.87 & 119 & 48.71 & 4215 & 979784.4 & -7.3 & -151.1 & 0.4 & 0.8 & -151.6 & -0.4 \\
\hline 04LOV056 & 40 & 12.64 & 119 & 48.12 & 4327 & 979777.7 & -3.2 & -150.8 & 0.2 & 0.7 & -151.4 & -0.2 \\
\hline 04LOV057 & 40 & 13.20 & 119 & 47.80 & 4630 & 979759.3 & 6.0 & -151.9 & 0.2 & 1.2 & -152.1 & -1.1 \\
\hline 04LOV058 & 40 & 13.12 & 119 & 47.46 & 4802 & 979746.4 & 9.5 & -154.3 & 0.3 & 1.9 & -153.8 & -2.8 \\
\hline 04LOV059 & 40 & 13.39 & 119 & 46.66 & 4703 & 979755.4 & 8.7 & -151.7 & 0.2 & 1.2 & -151.9 & -1.0 \\
\hline 04LOV060 & 40 & 13.02 & 119 & 46.31 & 4628 & 979760.1 & 6.9 & -150.9 & 0.2 & 0.8 & -151.5 & -0.6 \\
\hline 04LOV061 & 40 & 16.82 & 119 & 46.77 & 3889 & 979809.4 & -18.9 & -151.5 & 0.0 & 0.4 & -152.4 & -1.4 \\
\hline 04LOV062 & 40 & 16.75 & 119 & 45.99 & 3878 & 979808.1 & -21.1 & -153.4 & 0.0 & 0.3 & -154.3 & -3.3 \\
\hline 04LOV063 & 40 & 16.70 & 119 & 45.36 & 3867 & 979807.5 & -22.6 & -154.5 & 0.0 & 0.3 & -155.4 & -4.5 \\
\hline 04LOV064 & 40 & 17.07 & 119 & 45.67 & 3854 & 979808.0 & -24.0 & -155.4 & 0.0 & 0.3 & -156.3 & -5.3 \\
\hline 04LOV065 & 40 & 23.15 & 119 & 49.89 & 3964 & 979816.4 & -14.3 & -149.5 & 1.8 & 3.0 & -147.8 & 3.9 \\
\hline 04LOV066 & 40 & 23.16 & 119 & 49.72 & 3901 & 979816.1 & -20.5 & -153.5 & 0.3 & 1.5 & -153.3 & -1.7 \\
\hline 04LOV067 & 40 & 23.22 & 119 & 49.44 & 3854 & 979814.5 & -26.6 & -158.1 & 0.0 & 0.9 & -158.4 & -6.7 \\
\hline 04LOV068 & 40 & 23.28 & 119 & 49.16 & 3852 & 979812.8 & -28.6 & -159.9 & 0.0 & 0.6 & -160.6 & -8.9 \\
\hline 04LOV069 & 40 & 23.22 & 119 & 48.97 & 3868 & 979810.2 & -29.5 & -161.4 & 0.0 & 0.5 & -162.2 & -10.6 \\
\hline 04LOV070 & 40 & 23.22 & 119 & 48.64 & 3864 & 979808.9 & -31.2 & -163.0 & 0.0 & 0.4 & -163.9 & -12.3 \\
\hline 04LOV071 & 40 & 23.22 & 119 & 48.34 & 3866 & 979807.4 & -32.6 & -164.4 & 0.0 & 0.3 & -165.4 & -13.8 \\
\hline 04LOV072 & 40 & 23.25 & 119 & 48.04 & 3854 & 979806.1 & -35.0 & -166.5 & 0.0 & 0.3 & -167.4 & -15.9 \\
\hline 04LOV073 & 40 & 23.23 & 119 & 47.81 & 3852 & 979804.2 & -37.1 & -168.5 & 0.0 & 0.2 & -169.5 & -17.9 \\
\hline 04LOV074 & 40 & 23.18 & 119 & 47.55 & 3841 & 979803.5 & -38.7 & -169.7 & 0.0 & 0.2 & -170.7 & -19.2 \\
\hline 04LOV075 & 40 & 23.22 & 119 & 47.25 & 3835 & 979800.9 & -42.0 & -172.8 & 0.0 & 0.2 & -173.8 & -22.3 \\
\hline 04LOV076 & 40 & 23.22 & 119 & 46.98 & 3846 & 979799.4 & -42.4 & -173.6 & 0.0 & 0.2 & -174.7 & -23.1 \\
\hline 04LOV077 & 40 & 23.21 & 119 & 46.69 & 3845 & 979798.7 & -43.2 & -174.4 & 0.0 & 0.2 & -175.4 & -23.9 \\
\hline 04LOV078 & 40 & 23.21 & 119 & 46.42 & 3838 & 979798.2 & -44.4 & -175.3 & 0.0 & 0.2 & -176.4 & -24.9 \\
\hline 04LOV079 & 40 & 23.22 & 119 & 46.07 & 3847 & 979798.0 & -43.7 & -174.9 & 0.0 & 0.2 & -176.0 & -24.5 \\
\hline 04LOV080 & 40 & 23.22 & 119 & 45.69 & 3852 & 979798.2 & -43.1 & -174.5 & 0.0 & 0.2 & -175.5 & -24.1 \\
\hline 04LOV081 & 40 & 23.22 & 119 & 45.39 & 3849 & 979798.5 & -43.1 & -174.3 & 0.0 & 0.2 & -175.4 & -24.0 \\
\hline 04LOV082 & 40 & 23.23 & 119 & 45.10 & 3851 & 979799.0 & -42.4 & -173.7 & 0.0 & 0.2 & -174.8 & -23.3 \\
\hline 04LOV083 & 40 & 23.22 & 119 & 44.81 & 3852 & 979800.0 & -41.3 & -172.7 & 0.0 & 0.2 & -173.7 & -22.3 \\
\hline 04LOV084 & 40 & 23.22 & 119 & 44.53 & 3853 & 979801.0 & -40.2 & -171.6 & 0.0 & 0.2 & -172.7 & -21.3 \\
\hline 04LOV085 & 40 & 23.22 & 119 & 44.24 & 3855 & 979801.3 & -39.7 & -171.2 & 0.0 & 0.2 & -172.2 & -20.8 \\
\hline 04LOV086 & 40 & 23.22 & 119 & 43.94 & 3859 & 979801.5 & -39.1 & -170.7 & 0.0 & 0.3 & -171.7 & -20.3 \\
\hline 04LOV087 & 40 & 23.22 & 119 & 43.66 & 3856 & 979801.9 & -39.0 & -170.5 & 0.0 & 0.3 & -171.4 & -20.0 \\
\hline 04LOV088 & 40 & 23.21 & 119 & 43.37 & 3861 & 979802.5 & -37.9 & -169.6 & 0.0 & 0.3 & -170.5 & -19.2 \\
\hline 04LOV089 & 40 & 23.22 & 119 & 43.10 & 3858 & 979803.4 & -37.2 & -168.8 & 0.0 & 0.4 & -169.7 & -18.3 \\
\hline 04LOV090 & 40 & 23.22 & 119 & 42.81 & 3857 & 979804.9 & -35.9 & -167.4 & 0.0 & 0.4 & -168.2 & -16.9 \\
\hline 04LOV091 & 40 & 23.22 & 119 & 42.50 & 3864 & 979807.2 & -32.9 & -164.7 & 0.0 & 0.5 & -165.4 & -14.1 \\
\hline 04LOV092 & 40 & 23.22 & 119 & 42.22 & 3867 & 979810.2 & -29.7 & -161.6 & 0.0 & 0.6 & -162.2 & -10.9 \\
\hline
\end{tabular}




\begin{tabular}{|c|c|c|c|c|c|c|c|c|c|c|c|c|}
\hline 04LOV093 & 40 & 23.23 & 119 & 41.92 & 3877 & 979811.7 & -27.3 & -159.5 & 0.0 & 0.7 & -160.0 & -8.7 \\
\hline 04LOV094 & 40 & 23.21 & 119 & 41.67 & 3887 & 979812.7 & -25.2 & -157.8 & 0.0 & 0.8 & -158.2 & -6.9 \\
\hline 04LOV095 & 40 & 24.31 & 119 & 43.98 & 3841 & 979801.9 & -42.0 & -173.0 & 0.0 & 0.3 & -174.0 & -22.4 \\
\hline 04LOV096 & 40 & 25.13 & 119 & 44.01 & 3838 & 979803.5 & -42.0 & -172.9 & 0.0 & 0.3 & -173.9 & -22.2 \\
\hline 04LOV097 & 40 & 26.01 & 119 & 44.00 & 3837 & 979807.3 & -39.5 & -170.4 & 0.0 & 0.2 & -171.4 & -19.6 \\
\hline 04LOV098 & 40 & 26.02 & 119 & 45.13 & 3852 & 979803.6 & -41.9 & -173.3 & 0.0 & 0.1 & -174.3 & -22.5 \\
\hline 04LOV099 & 40 & 25.14 & 119 & 45.12 & 3842 & 979800.7 & -44.4 & -175.4 & 0.0 & 0.2 & -176.5 & -24.8 \\
\hline 04LOV100 & 40 & 24.26 & 119 & 45.15 & 3833 & 979799.5 & -45.1 & -175.8 & 0.0 & 0.2 & -176.8 & -25.3 \\
\hline 04LOV101 & 40 & 24.29 & 119 & 46.29 & 3854 & 979797.6 & -45.1 & -176.5 & 0.0 & 0.2 & -177.6 & -26.0 \\
\hline 04LOV102 & 40 & 25.14 & 119 & 46.28 & 3831 & 979800.2 & -46.0 & -176.6 & 0.0 & 0.2 & -177.7 & -25.9 \\
\hline 04LOV103 & 40 & 26.01 & 119 & 46.28 & 3849 & 979803.2 & -42.6 & -173.8 & 0.0 & 0.1 & -174.9 & -23.0 \\
\hline 04LOV104 & 40 & 26.01 & 119 & 47.37 & 3849 & 979802.6 & -43.1 & -174.3 & 0.0 & 0.2 & -175.4 & -23.4 \\
\hline 04LOV105 & 40 & 25.14 & 119 & 47.34 & 3849 & 979800.0 & -44.4 & -175.7 & 0.0 & 0.2 & -176.7 & -24.9 \\
\hline 04LOV106 & 40 & 24.27 & 119 & 47.36 & 3842 & 979799.3 & -44.5 & -175.5 & 0.0 & 0.2 & -176.6 & -24.9 \\
\hline 04LOV107 & 40 & 26.60 & 119 & 47.84 & 3877 & 02.9 & -41.0 & -173.3 & 0.0 & 0.2 & -174.3 & -22.2 \\
\hline 04LOV108 & 40 & 26.60 & 119 & 47.61 & 3837 & 979805.6 & -42.2 & -173.0 & 0.0 & 0.2 & -174.0 & -21.9 \\
\hline 04LOV109 & 40 & 26.61 & 119 & 47.37 & 3839 & 979805.7 & -41.9 & -172.8 & 0.0 & 0.2 & -173.8 & -21.7 \\
\hline 04LOV110 & 40 & 26.62 & 119 & 47.13 & 3842 & 979805.8 & -41.5 & -172.6 & 0.0 & 0.2 & -173.6 & -21.5 \\
\hline 04LOV111 & 40 & 26.62 & 119 & 46.88 & 3849 & 979806.0 & -40.7 & -171.9 & 0.0 & 0.1 & -173.0 & -20.9 \\
\hline 04LO & 40 & 26.62 & 119 & 46.68 & 3849 & 979 & -40.1 & -171.4 & 0.0 & 0.1 & -172.5 & -20.4 \\
\hline 04LO & 40 & 26.62 & 119 & 46.45 & 3851 & 979 & -39.0 & -170.4 & 0.0 & 0.1 & -171.5 & -19.4 \\
\hline 04LOV114 & 40 & 26.62 & 119 & 46.28 & 3851 & 9798 & -38.3 & -169.6 & 0.0 & 0.1 & -170.8 & -18.7 \\
\hline 04LOV115 & 40 & 26.63 & 119 & 46.00 & 3850 & 979809.5 & -37.1 & -168.4 & 0.0 & 0.1 & -169.5 & -17.5 \\
\hline 04LOV116 & 40 & 26.63 & 119 & 45.70 & 3849 & 979810.5 & -36.2 & -167.5 & 0.0 & 0.1 & -168.6 & -16.5 \\
\hline 04LOV117 & 40 & 26.61 & 119 & 45.37 & 3848 & 979809.2 & -37.5 & -168.7 & 0.0 & 0.1 & -169.8 & -17.8 \\
\hline 04LOV118 & 40 & 26.61 & 119 & 45.09 & 3849 & 06.3 & -40.3 & -171.5 & 0.0 & 0.1 & -172.6 & -20.7 \\
\hline 04LOV119 & 40 & 26.62 & 119 & 44.83 & 3850 & & -40.8 & -172.1 & 0.0 & 0.1 & -173.2 & -21.2 \\
\hline 04LOV120 & 40 & 26.61 & 119 & 44.51 & 3849 & 979806.6 & -40.0 & -171.2 & 0.0 & 0.1 & -172.3 & -20.4 \\
\hline 04LOV121 & 40 & 26.63 & 119 & 44.24 & 3852 & 979807.6 & -38.8 & -170.2 & 0.0 & 0.2 & -171.3 & -19.3 \\
\hline 04LOV122 & 40 & 26.62 & 119 & 43.98 & 3853 & 979808.7 & -37.5 & -168.9 & & 0.2 & -170.0 & -18.1 \\
\hline 04LOV123 & 40 & 26.62 & 119 & 43.70 & 3853 & 979809.6 & -36.7 & -168.1 & 0.0 & 0.2 & -169.1 & -17.2 \\
\hline 04LOV124 & 40 & 26.61 & 119 & 43.37 & 3854 & 979810.5 & -35.7 & -167.1 & 0.0 & 0.2 & -168.2 & -16.3 \\
\hline 04LOV125 & 40 & 26.61 & 119 & 43.08 & 3849 & 979811.1 & -35.5 & -166.8 & 0.0 & 0.2 & -167.8 & -15.9 \\
\hline 04LOV126 & 40 & 26.61 & 119 & 42.82 & 3847 & 979811.0 & -35.7 & -167.0 & 0.0 & 0.3 & -167.9 & -16.1 \\
\hline 04LOV127 & 40 & 26.61 & 119 & 42.54 & 3852 & 979810.2 & -36.2 & -167.5 & 0.0 & 0.3 & -168.5 & -16.7 \\
\hline 04LOV128 & 40 & 26.61 & 119 & 42.24 & 3855 & 979810.3 & -35.8 & -167.2 & 0.0 & 0.3 & -168.2 & -16.3 \\
\hline 04LOV129 & 40 & 28.64 & 119 & 41.77 & 3859 & 979810.8 & -38.0 & -169.6 & 0.0 & 0.2 & -170.5 & -18.4 \\
\hline 04LOV130 & 40 & 27.77 & 119 & 41.79 & 3857 & 979811.3 & -36.2 & -167.8 & 0.0 & 0.3 & -168.7 & -16.7 \\
\hline 04LOV131 & 40 & 26.62 & 119 & 42.03 & 3851 & 979810.5 & -35.9 & -167.2 & 0.0 & 0.4 & -168.1 & -16.3 \\
\hline 04LOV132 & 40 & 26.61 & 119 & 41.76 & 3854 & 979810.9 & -35.2 & -166.7 & 0.0 & 0.4 & -167.5 & -15.7 \\
\hline 04LOV133 & 40 & 26.62 & 119 & & 3858 & & -34.4 & -166.0 & 0.0 & 0.5 & -166.7 & -15.0 \\
\hline 04LOV134 & 40 & 26.61 & 119 & 41.17 & 3857 & 979812.0 & -33.9 & -165.4 & 0.0 & 0.5 & -166.2 & -14.4 \\
\hline 04LOV135 & 40 & 26.61 & 119 & 40.90 & 3862 & 979812.9 & -32.4 & -164.1 & 0.0 & 0.6 & -164.8 & -13.0 \\
\hline 04LOV136 & 40 & 26.62 & 119 & 40.60 & 3865 & 979814.1 & -31.0 & -162.8 & 0.0 & 0.7 & -163.3 & -11.6 \\
\hline 04LOV137 & 40 & 26.62 & 119 & 40.32 & 3872 & 979815.0 & -29.4 & -161.5 & 0.0 & 0.8 & -161.9 & -10.2 \\
\hline 04LOV138 & 40 & 30.02 & 119 & 45.21 & 3845 & 979811.4 & -40.6 & -171.8 & 0.0 & 0.1 & -172.9 & -20.1 \\
\hline 04LOV139 & 40 & 30.01 & 119 & 44.98 & 3845 & 979810.5 & -41.5 & -172.6 & 0.0 & 0.1 & -173.8 & -21.0 \\
\hline 04LOV140 & 40 & 30.01 & 119 & 44.74 & 3840 & 979809.7 & -42.8 & -173.8 & 0.0 & 0.1 & -174.9 & -22.1 \\
\hline 04LOV141 & 40 & 30.01 & 119 & 44.42 & 3842 & 979809.0 & -43.3 & -174.4 & 0.0 & 0.1 & -175.5 & -22.8 \\
\hline 04LOV142 & 40 & 30.02 & 119 & 44.08 & 3843 & 979808.9 & -43.3 & -174.4 & 0.0 & 0.1 & -175.5 & -22.8 \\
\hline
\end{tabular}




\begin{tabular}{|c|c|c|c|c|c|c|c|c|c|c|c|c|}
\hline 04LOV143 & 40 & 30.01 & 119 & 43.81 & 3849 & 979809.0 & -42.7 & -173.9 & 0.0 & 0.1 & -175.1 & -22.4 \\
\hline 04LOV144 & 40 & 30.01 & 119 & 43.50 & 3848 & 979809.0 & -42.8 & -174.0 & 0.0 & 0.1 & -175.2 & -22.5 \\
\hline 04LOV145 & 40 & 30.01 & 119 & 43.22 & 3851 & 979809.5 & -42.0 & -173.4 & 0.0 & 0.1 & -174.5 & -21.8 \\
\hline 04LOV146 & 40 & 30.02 & 119 & 42.95 & 3851 & 979809.7 & -41.8 & -173.1 & 0.0 & 0.1 & -174.2 & -21.6 \\
\hline 04LOV147 & 40 & 30.02 & 119 & 42.65 & 3846 & 979810.1 & -41.8 & -173.0 & 0.0 & 0.2 & -174.1 & -21.5 \\
\hline 04LOV148 & 40 & 30.02 & 119 & 42.38 & 3848 & 979810.7 & -41.1 & -172.3 & 0.0 & 0.2 & -173.4 & -20.9 \\
\hline 04LOV149 & 40 & 30.01 & 119 & 42.11 & 3849 & 979811.3 & -40.3 & -171.6 & 0.0 & 0.2 & -172.6 & -20.1 \\
\hline 04LOV150 & 40 & 30.02 & 119 & 41.80 & 3852 & 979812.2 & -39.2 & -170.6 & 0.0 & 0.2 & -171.6 & -19.1 \\
\hline 04LOV151 & 40 & 30.00 & 119 & 41.51 & 3853 & 979812.2 & -39.1 & -170.5 & 0.0 & 0.2 & -171.5 & -19.0 \\
\hline 04LOV152 & 40 & 29.99 & 119 & 41.24 & 3851 & 979812.4 & -39.1 & -170.4 & 0.0 & 0.2 & -171.4 & -18.9 \\
\hline 04LOV153 & 40 & 30.01 & 119 & 40.95 & 3853 & 979812.9 & -38.4 & -169.8 & 0.0 & 0.3 & -170.7 & -18.3 \\
\hline 04LOV154 & 40 & 30.01 & 119 & 40.65 & 3851 & 979813.6 & -37.9 & -169.2 & 0.0 & 0.3 & -170.1 & -17.7 \\
\hline 04LOV155 & 40 & 30.01 & 119 & 40.36 & 3852 & 979814.0 & -37.4 & & 0.0 & 0.3 & -169.6 & -17.2 \\
\hline 04LOV156 & 40 & 30.01 & 119 & 40.05 & 3858 & 979814.3 & -36.5 & -168.1 & 0.0 & 0.4 & -168.9 & -16.5 \\
\hline 04LOV157 & 40 & 30.02 & 119 & 39.75 & 3857 & 979814.8 & -36.1 & -167.7 & 0.0 & 0.5 & -168.5 & -16.1 \\
\hline 04LOV158 & 40 & 30.01 & 119 & 39.46 & 3857 & 979815.5 & -35.4 & -167.0 & 0.0 & 0.5 & -167.7 & -15.3 \\
\hline 04LOV159 & 40 & 30.01 & 119 & 39.00 & 3862 & 979817.4 & -33.1 & -164.8 & 0.0 & 0.7 & -165.3 & -13.0 \\
\hline 04LOV160 & 40 & 30.16 & 119 & 39.18 & 3849 & 979816.3 & -35.5 & -166.8 & 0.0 & 0.6 & -167.4 & -15.0 \\
\hline 04LOV161 & 40 & 30.33 & 119 & 39.37 & 3838 & 979815.4 & -37.8 & -168.7 & 0.0 & 0.6 & -169.3 & -16.9 \\
\hline $04 \mathrm{LO}$ & 40 & 30.51 & 119 & 39.60 & 3837 & 979814.5 & -39.1 & -169.9 & 0.0 & 0.5 & -170.6 & -18.1 \\
\hline 04LOV163 & 40 & 30.68 & 119 & 39.79 & 3855 & 979813.9 & -38.2 & -169.6 & 0.0 & 0.4 & -170.5 & -17.9 \\
\hline 04LOV164 & 40 & 30.85 & 119 & 39.96 & 3838 & 979813.3 & -40.6 & -171.5 & 0.0 & 0.4 & -172.3 & -19.8 \\
\hline 04LOV165 & 40 & 31.01 & 119 & 40.17 & 3845 & 979813.0 & -40.5 & -171.6 & 0.0 & 0.3 & -172.5 & -19.9 \\
\hline 04LOV166 & 40 & 31.21 & 119 & 40.37 & 3849 & 979813.0 & -40.4 & -171.7 & 0.0 & 0.3 & -172.6 & -19.9 \\
\hline 04LOV167 & 40 & 31.38 & 119 & 40.61 & 3848 & 979813.4 & -40.4 & -171.6 & 0.0 & 0.2 & -172.6 & -19.9 \\
\hline 04LOV168 & 40 & 31.56 & 119 & 40.79 & 3836 & 314.2 & -41.0 & -171.9 & 0.0 & 0.2 & -172.8 & -20.0 \\
\hline 04LOV169 & 40 & 31.72 & 119 & 41.00 & 3858 & 979815.1 & -38.3 & -169.9 & 0.0 & 0.2 & -170.9 & -18.0 \\
\hline 04LOV170 & 40 & 31.87 & 119 & 41.18 & 3823 & 979815.7 & -41.1 & -171.5 & 0.0 & 0.3 & -172.5 & -19.5 \\
\hline 04LOV171 & 40 & 32.01 & 119 & 41.42 & 3827 & 979815.3 & -41.5 & -172.0 & 0.0 & 0.2 & -173.0 & -20.0 \\
\hline 04LOV172 & 40 & 32.21 & 119 & 41.60 & 3833 & 979814.7 & -41.7 & -172.5 & & 0.2 & -173.5 & -20.4 \\
\hline 04LOV173 & 40 & 32.38 & 119 & 41.80 & 3838 & 979814.2 & -42.0 & -172.9 & 0.0 & 0.2 & -174.0 & -20.8 \\
\hline 04LOV174 & 40 & 32.50 & 119 & 42.01 & 3840 & 979814.1 & -42.1 & -173.1 & 0.0 & 0.2 & -174.2 & -21.0 \\
\hline 04LOV175 & 40 & 32.66 & 119 & 42.16 & 3838 & 979815.2 & -41.5 & -172.4 & 0.0 & 0.2 & -173.4 & -20.2 \\
\hline 04LOV176 & 40 & 32.85 & 119 & 42.31 & 3838 & 979815.2 & -41.7 & -172.6 & 0.0 & 0.2 & -173.6 & -20.3 \\
\hline 04LOV177 & 40 & 32.99 & 119 & 42.52 & 3843 & 979816.5 & -40.1 & -171.2 & 0.0 & 0.1 & -172.3 & -18.9 \\
\hline 04LOV178 & 40 & 33.13 & 119 & 42.74 & 3848 & 979818.1 & -38.3 & -169.5 & 0.0 & 0.1 & -170.6 & -17.2 \\
\hline 04LOV179 & 40 & 33.00 & 119 & 43.03 & 3846 & 979817.8 & -38.6 & -169.8 & 0.0 & 0.1 & -170.9 & -17.4 \\
\hline 04LOV180 & 40 & 33.01 & 119 & 42.22 & 3845 & 979815.9 & -40.6 & -171.8 & 0.0 & 0.2 & -172.8 & -19.5 \\
\hline 04LOV181 & 40 & 33.00 & 119 & 41.89 & 3843 & 979815.7 & -40.9 & -172.0 & 0.0 & 0.2 & -173.1 & -19.7 \\
\hline 04LOV182 & 40 & 33.02 & 119 & 41.58 & 3844 & 979816.3 & -40.3 & -171.4 & 0.0 & 0.2 & -172.4 & -19.1 \\
\hline 04LOV183 & 40 & 33.01 & 119 & 41.30 & 3843 & 979817.0 & -39.7 & -170.8 & 0.0 & 0.2 & -171.8 & -18.5 \\
\hline 04LOV184 & 40 & 33.02 & 119 & 41.01 & 3842 & 979817.8 & -39.0 & -170.0 & 0.0 & 0.2 & -171.0 & -17.7 \\
\hline 04LOV185 & 40 & 33.00 & 119 & 40.71 & 3845 & 979818.3 & -38.1 & -169.3 & 0.0 & 0.2 & -170.3 & -17.1 \\
\hline 04LOV186 & 40 & 33.01 & 119 & 40.41 & 3848 & 979819.0 & -37.2 & -168.5 & 0.0 & 0.2 & -169.5 & -16.3 \\
\hline 04LOV187 & 40 & 33.01 & 119 & 40.14 & 3848 & 979819.6 & -36.6 & -167.9 & 0.0 & 0.2 & -168.9 & -15.7 \\
\hline 04LOV188 & 40 & 33.01 & 119 & 39.84 & 3851 & 979819.6 & -36.4 & -167.7 & 0.0 & 0.2 & -168.7 & -15.5 \\
\hline 04LOV189 & 40 & 33.01 & 119 & 39.53 & 3852 & 979819.0 & -36.8 & -168.2 & 0.0 & 0.2 & -169.2 & -16.1 \\
\hline 04LOV190 & 40 & 33.01 & 119 & 39.22 & 3853 & 979818.9 & -36.9 & -168.3 & 0.0 & 0.3 & -169.2 & -16.1 \\
\hline 04LOV191 & 40 & 33.01 & 119 & 38.91 & 3853 & 979819.1 & -36.7 & -168.1 & 0.0 & 0.3 & -169.0 & -15.9 \\
\hline 04LOV192 & 40 & 33.02 & 119 & 38.60 & 3850 & 979818.7 & -37.3 & -168.6 & 0.0 & 0.3 & -169.5 & -16.5 \\
\hline
\end{tabular}




\begin{tabular}{|c|c|c|c|c|c|c|c|c|c|c|c|c|}
\hline 04LOV193 & 40 & 33.01 & 119 & 38.34 & 3851 & 979818.6 & -37.3 & -168.7 & 0.0 & 0.4 & -169.5 & -16.5 \\
\hline 04LOV194 & 40 & 33.01 & 119 & 38.05 & 3850 & 979818.8 & -37.2 & -168.6 & 0.0 & 0.4 & -169.4 & -16.4 \\
\hline 04LOV195 & 40 & 33.01 & 119 & 37.75 & 3853 & 979818.9 & -36.9 & -168.3 & 0.0 & 0.5 & -169.1 & -16.1 \\
\hline 04LOV196 & 40 & 33.01 & 119 & 37.43 & 3852 & 979818.8 & -37.0 & -168.4 & 0.0 & 0.5 & -169.1 & -16.1 \\
\hline 04LOV197 & 40 & 33.01 & 119 & 37.17 & 3853 & 979819.1 & -36.7 & -168.1 & 0.0 & 0.6 & -168.8 & -15.8 \\
\hline 04LOV198 & 40 & 33.02 & 119 & 36.86 & 3857 & 979819.4 & -36.0 & -167.5 & 0.0 & 0.6 & -168.1 & -15.2 \\
\hline 04LOV199 & 40 & 33.00 & 119 & 36.56 & 3862 & 979820.2 & -34.7 & -166.4 & 0.0 & 0.7 & -167.0 & -14.1 \\
\hline 04LOV200 & 40 & 33.02 & 119 & 36.32 & 3855 & 979821.1 & -34.5 & -165.9 & 0.0 & 0.8 & -166.3 & -13.5 \\
\hline 04LOV201 & 40 & 17.67 & 119 & 45.16 & 3857 & 979806.4 & -26.2 & -157.7 & 0.0 & 0.2 & -158.7 & -7.7 \\
\hline 04LOV202 & 40 & 18.18 & 119 & 45.17 & 3852 & 979806.8 & -27.0 & -158.4 & 0.0 & 0.2 & -159.4 & -8.4 \\
\hline 04LOV203 & 40 & 19.04 & 119 & 45.15 & 3851 & 979807.5 & -27.6 & -159.0 & 0.0 & 0.2 & -160.0 & -9.0 \\
\hline 04LOV204 & 40 & 19.94 & 119 & 45.14 & 3849 & 979806.1 & -30.6 & -161.9 & 0.0 & 0.2 & -162.9 & -11.8 \\
\hline 04LOV205 & 40 & 20.80 & 119 & 45.14 & 3852 & 979805.3 & -32.4 & -163.8 & 0.0 & 0.2 & -164.8 & -13.7 \\
\hline 04LOV206 & 40 & 21.65 & 119 & 45.13 & 3848 & 979802.4 & -36.9 & -168.1 & 0.0 & 0.2 & -169.2 & -17.9 \\
\hline 04LOV207 & 40 & 38.20 & 119 & 27.47 & 3894 & 979832.4 & -27.2 & -160.0 & 0.0 & 0.3 & -161.0 & -7.4 \\
\hline 04LOV208 & 40 & 39.11 & 119 & 29.01 & 3903 & 979835.7 & -24.5 & -157.6 & 0.0 & 0.3 & -158.5 & -4.6 \\
\hline 04LOV209 & 40 & 39.34 & 119 & 29.05 & 3882 & 979835.5 & -26.9 & -159.4 & 0.0 & 0.3 & -160.3 & -6.2 \\
\hline 04LOV210 & 40 & 39.56 & 119 & 29.06 & 3874 & 979834.4 & -29.2 & -161.3 & 0.0 & 0.3 & -162.3 & -8.1 \\
\hline 04LOV211 & 40 & 39.80 & 119 & 29.04 & 3854 & 979832.0 & -33.8 & -165.3 & 0.0 & 0.4 & -166.1 & -11.9 \\
\hline 04LOV212 & 40 & 40.04 & 119 & 29.05 & 3862 & 979831.0 & -34.3 & -166.1 & 0.0 & 0.4 & -166.9 & -12.6 \\
\hline 04LOV213 & 40 & 40.29 & 119 & 29.06 & 3867 & 979830.5 & -34.8 & -166.7 & 0.0 & 0.4 & -167.5 & -13.2 \\
\hline 04LOV214 & 40 & 40.53 & 119 & 29.05 & 3842 & 979829.7 & -38.3 & -169.4 & 0.0 & 0.5 & -170.1 & -15.7 \\
\hline 04LOV215 & 40 & 40.77 & 119 & 29.06 & 3854 & 979829.1 & -38.2 & -169.6 & 0.0 & 0.5 & -170.4 & -15.9 \\
\hline 04LOV216 & 40 & 41.00 & 119 & 29.05 & 3853 & 979829.0 & -38.6 & -170.1 & 0.0 & 0.6 & -170.7 & -16.1 \\
\hline 04LOV217 & 40 & 21.22 & 119 & 40.47 & 4319 & 979789.1 & -5.3 & -152.6 & 0.4 & 1.6 & -152.3 & -1.3 \\
\hline 04LOV218 & 40 & 22.00 & 119 & 39.29 & 4810 & 979767.1 & 17.7 & -146.4 & 0.9 & 3.9 & -143.9 & 7.0 \\
\hline 04LOV219 & 40 & 22.88 & 119 & 39.86 & 4452 & 979786.8 & 2.5 & -149.4 & 0.1 & 2.7 & -148.0 & 3.1 \\
\hline 04LOV220 & 40 & 23.76 & 119 & 39.46 & 4618 & 979777.4 & 7.3 & -150.2 & 0.7 & 4.1 & -147.5 & 3.6 \\
\hline 04LOV221 & 40 & 25.10 & 119 & 39.51 & 4239 & 979801.4 & -6.3 & -150.9 & 1.0 & 3.4 & -148.8 & 2.5 \\
\hline 04LOV222 & 40 & 14.02 & 119 & 40.26 & 5175 & 979722.7 & 19.5 & -157.0 & 0.3 & 3.1 & -155.3 & -4.7 \\
\hline 04LOV223 & 40 & 17.60 & 119 & 42.05 & 4790 & 979752.2 & 7.4 & -156.0 & 1.3 & 5.0 & -152.4 & -1.6 \\
\hline 04LOV224 & 40 & 50.48 & 119 & 26.34 & 7555 & 979627.8 & 93.9 & -163.8 & 0.3 & 10.7 & -154.6 & 2.0 \\
\hline 04LOV225 & 40 & 47.04 & 119 & 24.75 & 8214 & 979569.0 & 102.2 & -178.0 & 0.2 & 19.3 & -160.1 & -5.4 \\
\hline 04LOV226 & 40 & 44.89 & 119 & 22.28 & 5247 & 979764.5 & 22.1 & -156.8 & 0.1 & 5.8 & -152.5 & 1.9 \\
\hline 04LOV227 & 40 & 43.51 & 119 & 22.46 & 5248 & 979761.2 & 20.8 & -158.1 & 0.2 & 5.0 & -154.6 & -0.5 \\
\hline 04LOV228 & 40 & 20.06 & 119 & 41.77 & 4641 & 979760.0 & -2.4 & -160.7 & 2.6 & 5.5 & -156.6 & -5.7 \\
\hline 04LOV229 & 40 & 11.55 & 119 & 45.10 & 4035 & 979794.4 & -12.3 & -149.9 & 0.1 & 1.1 & -150.1 & 1.1 \\
\hline 04LOV230 & 40 & 12.03 & 119 & 45.07 & 4166 & 979787.3 & -7.8 & -149.9 & 0.7 & 1.6 & -149.6 & 1.5 \\
\hline 04LOV231 & 40 & 12.57 & 119 & 45.15 & 4352 & 979776.8 & -1.6 & -150.1 & 0.7 & 1.6 & -149.8 & 1.2 \\
\hline 04LOV232 & 40 & 12.89 & 119 & 45.32 & 4452 & 979772.2 & 2.6 & -149.2 & 0.6 & 1.5 & -149.1 & 1.9 \\
\hline 04LOV233 & 40 & 41.46 & 119 & 29.05 & 3875 & 979829.2 & -37.1 & -169.2 & 0.0 & 0.6 & -169.8 & -15.1 \\
\hline 04LOV234 & 40 & 41.67 & 119 & 29.06 & 3876 & 979828.4 & -38.2 & -170.4 & 0.0 & 0.7 & -170.9 & -16.1 \\
\hline 04LOV235 & 40 & 41.23 & 119 & 29.06 & 3856 & 979829.5 & -38.2 & -169.8 & 0.0 & 0.6 & -170.4 & -15.7 \\
\hline 04LOV236 & 40 & 40.62 & 119 & 35.69 & 3843 & 979843.8 & -24.2 & -155.3 & 0.0 & 1.8 & -154.7 & 0.7 \\
\hline 04LOV237 & 40 & 40.63 & 119 & 35.34 & 3847 & 979842.9 & -24.7 & -155.9 & 0.0 & 1.5 & -155.7 & -0.4 \\
\hline 04LOV238 & 40 & 40.62 & 119 & 35.00 & 3852 & 979841.4 & -25.9 & -157.2 & 0.0 & 1.3 & -157.2 & -1.9 \\
\hline 04LOV239 & 40 & 40.63 & 119 & 34.64 & 3852 & 979839.5 & -27.7 & -159.1 & 0.0 & 1.1 & -159.2 & -4.0 \\
\hline 04LOV240 & 40 & 40.63 & 119 & 34.25 & 3846 & 979837.5 & -30.3 & -161.5 & 0.0 & 1.0 & -161.8 & -6.6 \\
\hline 04LOV241 & 40 & 40.63 & 119 & 33.98 & 3846 & 979835.8 & -31.9 & -163.1 & 0.0 & 0.9 & -163.4 & -8.3 \\
\hline 04LOV242 & 40 & 40.62 & 119 & 33.65 & 3847 & 979833.8 & -33.8 & -165.0 & 0.0 & 0.8 & -165.5 & -10.4 \\
\hline
\end{tabular}




\begin{tabular}{|c|c|c|c|c|c|c|c|c|c|c|c|c|}
\hline $04 \mathrm{LOV} 243$ & 40 & 40.62 & 119 & 33.38 & 3849 & 979832.7 & -34.8 & -166.1 & 0.0 & 0.7 & -166.6 & -11.6 \\
\hline 04LOV244 & 40 & 40.63 & 119 & 33.07 & 3849 & 979831.9 & -35.6 & -166.9 & 0.0 & 0.7 & -167.5 & -12.5 \\
\hline 04LOV245 & 40 & 40.62 & 119 & 32.81 & 3849 & 979831.4 & -36.1 & -167.4 & 0.0 & 0.6 & -168.0 & -13.1 \\
\hline 04LOV246 & 40 & 40.63 & 119 & 32.51 & 3856 & 979831.1 & -35.7 & -167.2 & 0.0 & 0.6 & -167.9 & -13.0 \\
\hline 04LOV247 & 40 & 40.63 & 119 & 32.22 & 3854 & 979831.2 & -35.8 & -167.2 & 0.0 & 0.6 & -167.9 & -13.0 \\
\hline 04LOV248 & 40 & 40.62 & 119 & 31.90 & 3851 & 979830.8 & -36.5 & -167.8 & 0.0 & 0.5 & -168.5 & -13.7 \\
\hline 04LOV249 & 40 & 40.63 & 119 & 31.60 & 3854 & 979830.1 & -36.9 & -168.4 & 0.0 & 0.5 & -169.1 & -14.3 \\
\hline 04LOV250 & 40 & 40.63 & 119 & 31.28 & 3857 & 979830.9 & -35.9 & -167.4 & 0.0 & 0.5 & -168.2 & -13.4 \\
\hline 04LOV251 & 40 & 40.63 & 119 & 30.96 & 3859 & 979833.3 & -33.2 & -164.8 & 0.0 & 0.5 & -165.6 & -10.9 \\
\hline 04LOV252 & 40 & 40.63 & 119 & 30.61 & 3860 & 979833.4 & -33.1 & -164.7 & 0.0 & 0.5 & -165.5 & -10.9 \\
\hline 04LOV253 & 40 & 40.62 & 119 & 30.31 & 3860 & 979831.9 & -34.6 & -166.2 & 0.0 & 0.5 & -167.0 & -12.4 \\
\hline 04LOV254 & 40 & 40.62 & 119 & 29.94 & 3855 & 979830.8 & -36.2 & -167.6 & 0.0 & 0.5 & -168.4 & -13.9 \\
\hline 04LOV255 & 40 & 40.63 & 119 & 29.60 & 3853 & 979830.5 & -36.6 & -168.0 & 0.0 & 0.5 & -168.8 & -14.3 \\
\hline 04LOV256 & 40 & 40.63 & 119 & 29.22 & 3862 & 979829.8 & -36.5 & -168.2 & 0.0 & 0.5 & -168.9 & -14.5 \\
\hline 04LOV257 & 40 & 40.66 & 119 & 28.76 & 3864 & 9798 & -37.5 & -169.3 & 0.0 & 0.5 & -170.0 & -15.6 \\
\hline 04LOV258 & 40 & 40.61 & 119 & 28.39 & 3863 & 979828.9 & -37.2 & -169.0 & 0.0 & 0.5 & -169.7 & -15.4 \\
\hline 04LOV259 & 40 & 40.62 & 119 & 27.94 & 3860 & 979833.1 & -33.4 & -165.0 & 0.0 & 0.5 & -165.7 & -11.5 \\
\hline 04LOV260 & 40 & 40.62 & 119 & 27.68 & 3861 & 979833.2 & -33.1 & -164.8 & 0.0 & 0.6 & -165.5 & -11.2 \\
\hline 04LOV261 & 40 & 40.63 & 119 & 27.29 & 3860 & 979830.3 & -36.1 & -167.8 & 0.0 & 0.6 & -168.4 & -14.3 \\
\hline 262 & 40 & 40.62 & 119 & 26.93 & 3862 & 979 & -38.7 & -170.4 & 0.0 & 0.6 & -171.0 & -16.9 \\
\hline V263 & 40 & 40.62 & 119 & 26.46 & 3863 & 979 & -41.4 & -173.1 & 0.0 & 0.6 & -173.7 & -19.6 \\
\hline 04LOV264 & 40 & 40.62 & 119 & 26.10 & 3874 & 979822.8 & -42.4 & -174.5 & 0.0 & 0.7 & -175.0 & -21.0 \\
\hline 04LOV265 & 40 & 40.62 & 119 & 25.53 & 3892 & 979819.7 & -43.7 & -176.4 & 0.0 & 0.7 & -176.9 & -23.0 \\
\hline 04LOV266 & 40 & 40.63 & 119 & 25.22 & 3896 & 979819.0 & -44.1 & -176.9 & 0.0 & 0.8 & -177.4 & -23.4 \\
\hline 04LOV267 & 40 & 35.74 & 119 & 33.07 & 3847 & 979825.5 & -34.9 & -166.1 & 0.0 & 0.5 & -166.9 & -13.5 \\
\hline 04LOV268 & 40 & 35.73 & 119 & 33.34 & 3846 & 9798 & -35.2 & -166.4 & 0.0 & 0.5 & -167.1 & -13.8 \\
\hline 04LOV269 & 40 & 35.75 & 119 & 33.64 & 3844 & 979824.7 & -36.0 & -167.1 & 0.0 & 0.4 & -167.9 & -14.5 \\
\hline 04LOV270 & 40 & 35.74 & 119 & 33.95 & 3843 & 979824.6 & -36.1 & -167.2 & 0.0 & 0.4 & -168.0 & -14.6 \\
\hline 04LOV271 & 40 & 35.74 & 119 & 34.27 & 3844 & 979824.9 & -35.8 & -166.9 & 0.0 & 0.4 & -167.8 & -14.3 \\
\hline 04LOV272 & 40 & 35.75 & 119 & 34.58 & 3842 & 979825.4 & -35.5 & -166.5 & 0.0 & 0.4 & -167.4 & -13.9 \\
\hline 04LC & 40 & 35.74 & 119 & 34.92 & 3842 & 9798 & -35.1 & -166.2 & 0.0 & 0.4 & -167.0 & -13.5 \\
\hline 04LOV274 & 40 & 35.74 & 119 & 35.24 & 3841 & 979826.0 & -34.9 & -165.9 & 0.0 & 0.3 & -166.8 & -13.3 \\
\hline $04 \mathrm{LOV} 275$ & 40 & 35.75 & 119 & 35.57 & 3840 & 979826.1 & -34.9 & -165.9 & 0.0 & 0.3 & -166.8 & -13.2 \\
\hline 04LOV276 & 40 & 35.74 & 119 & 35.91 & 3843 & 979826.6 & -34.1 & -165.2 & 0.0 & 0.3 & -166.1 & -12.5 \\
\hline 04LOV277 & 40 & 35.75 & 119 & 36.24 & 3843 & 979827.6 & -33.2 & -164.3 & 0.0 & 0.3 & -165.2 & -11.5 \\
\hline 04LOV278 & 40 & 35.74 & 119 & 36.59 & 3843 & 979829.1 & -31.7 & -162.7 & 0.0 & 0.3 & -163.7 & -10.0 \\
\hline 04LOV279 & 40 & 35.75 & 119 & 36.91 & 3848 & 979830.0 & -30.3 & -161.6 & 0.0 & 0.3 & -162.5 & -8.8 \\
\hline 04LOV280 & 40 & 35.74 & 119 & 37.27 & 3845 & 979828.7 & -31.8 & -163.0 & 0.0 & 0.3 & -163.9 & -10.1 \\
\hline 04LOV281 & 40 & 35.74 & 119 & 37.58 & 3849 & 979827.9 & -32.4 & -163.6 & 0.0 & 0.3 & -164.5 & -10.8 \\
\hline 04LOV282 & 40 & 35.74 & 119 & 37.93 & 3850 & 979828.2 & -31.9 & -163.2 & 0.0 & 0.3 & -164.1 & -10.3 \\
\hline 04LOV283 & 40 & 35.73 & 11 & 38.23 & 3851 & 97982 & -30.4 & -161.7 & 0.0 & 0.3 & -162.6 & -8.8 \\
\hline 04LOV284 & 40 & 35.72 & 119 & 38.56 & 3847 & 979828.7 & -31.6 & -162.8 & 0.0 & 0.3 & -163.7 & -9.8 \\
\hline 04LOV285 & 40 & 35.73 & 119 & 38.89 & 3849 & 979828.2 & -32.0 & -163.3 & 0.0 & 0.3 & -164.2 & -10.3 \\
\hline 04LOV286 & 40 & 35.71 & 119 & 39.19 & 3849 & 979827.4 & -32.8 & -164.1 & 0.0 & 0.3 & -165.0 & -11.0 \\
\hline 04LOV287 & 40 & 35.71 & 119 & 39.52 & 3851 & 979827.7 & -32.2 & -163.6 & 0.0 & 0.3 & -164.5 & -10.5 \\
\hline 04LOV288 & 40 & 35.73 & 119 & 39.86 & 3849 & 979828.8 & -31.4 & -162.7 & 0.0 & 0.3 & -163.6 & -9.5 \\
\hline 04LOV289 & 40 & 35.55 & 119 & 39.63 & 3846 & 979827.5 & -32.7 & -163.9 & 0.0 & 0.3 & -164.8 & -10.9 \\
\hline 04LOV290 & 40 & 35.37 & 119 & 39.43 & 3846 & 979826.3 & -33.6 & -164.8 & 0.0 & 0.3 & -165.7 & -11.9 \\
\hline 04LOV291 & 40 & 35.22 & 119 & 39.21 & 3845 & 979824.9 & -34.8 & -166.0 & 0.0 & 0.3 & -166.9 & -13.2 \\
\hline 04LOV292 & 40 & 35.04 & 119 & 39.00 & 3842 & 979824.0 & -35.8 & -166.8 & 0.0 & 0.3 & -167.8 & -14.1 \\
\hline
\end{tabular}




\begin{tabular}{|c|c|c|c|c|c|c|c|c|c|c|c|c|}
\hline 04LOV293 & 40 & 34.89 & 119 & 38.79 & 3839 & 979823.6 & -36.2 & -167.2 & 0.0 & 0.3 & -168.1 & -14.5 \\
\hline 04LOV294 & 40 & 34.71 & 119 & 38.57 & 3840 & 979823.1 & -36.4 & -167.4 & 0.0 & 0.3 & -168.3 & -14.8 \\
\hline 04LOV295 & 40 & 34.54 & 119 & 38.31 & 3841 & 979821.8 & -37.4 & -168.4 & 0.0 & 0.3 & -169.3 & -15.9 \\
\hline 04LOV296 & 40 & 34.36 & 119 & 38.12 & 3843 & 979821.1 & -37.7 & -168.7 & 0.0 & 0.3 & -169.7 & -16.3 \\
\hline 04LOV297 & 40 & 34.21 & 119 & 37.90 & 3841 & 979820.5 & -38.1 & -169.2 & 0.0 & 0.3 & -170.1 & -16.7 \\
\hline 04LOV298 & 40 & 34.03 & 119 & 37.68 & 3840 & 979820.1 & -38.4 & -169.4 & 0.0 & 0.3 & -170.3 & -17.0 \\
\hline 04LOV299 & 40 & 33.88 & 119 & 37.44 & 3842 & 979819.7 & -38.4 & -169.4 & 0.0 & 0.4 & -170.3 & -17.1 \\
\hline 04LOV300 & 40 & 33.71 & 119 & 37.26 & 3848 & 979819.5 & -37.8 & -169.1 & 0.0 & 0.4 & -169.9 & -16.7 \\
\hline 04LOV301 & 40 & 33.55 & 119 & 37.04 & 3852 & 979819.5 & -37.2 & -168.6 & 0.0 & 0.5 & -169.4 & -16.3 \\
\hline 04LOV302 & 40 & 33.37 & 119 & 36.81 & 3851 & 979819.4 & -37.0 & -168.4 & 0.0 & 0.6 & -169.0 & -16.0 \\
\hline 04LOV303 & 40 & 33.16 & 119 & 36.51 & 3851 & 979820.1 & -36.0 & -167.4 & 0.0 & 0.7 & -167.9 & -15.0 \\
\hline 04LOV304 & 40 & 32.33 & 119 & 37.81 & 3841 & 979818.6 & -37.4 & -168.3 & 0.0 & 0.6 & -168.9 & -16.1 \\
\hline 04LOV305 & 40 & 27.67 & 119 & 50.87 & 3920 & 979812.8 & -28.8 & -162.5 & 0.0 & 0.6 & -163.1 & -10.5 \\
\hline 04LOV306 & 40 & 28.04 & 119 & 50.96 & 3928 & 979813.1 & -28.2 & -162.1 & 0.0 & 0.5 & -162.8 & -10.1 \\
\hline 04LOV307 & 40 & 24.00 & 119 & 49.70 & 3892 & 979815.6 & -23.0 & -155.8 & 0.0 & 0.6 & -156.4 & -4.6 \\
\hline 04LOV308 & 40 & 29.31 & 119 & 50.59 & 3927 & 979820.0 & -23.4 & -157.3 & 0.0 & 0.4 & -158.1 & -5.1 \\
\hline 04LOV309 & 40 & 30.08 & 119 & 52.21 & 3990 & 979815.3 & -23.2 & -159.3 & 0.0 & 0.8 & -159.8 & -6.3 \\
\hline 04LOV310 & 40 & 29.50 & 119 & 52.37 & 3984 & 979816.1 & -22.1 & -158.0 & 0.4 & 1.3 & -158.0 & -4.7 \\
\hline 04LOV311 & 40 & 28.42 & 119 & 53.36 & 4340 & 979799.8 & -3.4 & -151.4 & 0.1 & 0.9 & -151.8 & 1.1 \\
\hline 04LOV312 & 40 & 28.10 & 119 & 54.20 & 4372 & 979799.9 & 0.2 & -148.9 & 0.1 & 1.5 & -148.7 & 4.3 \\
\hline 04LOV313 & 40 & 27.98 & 119 & 54.72 & 4536 & 979789.7 & 5.6 & -149.1 & 0.3 & 2.1 & -148.4 & 4.6 \\
\hline 04LOV314 & 40 & 28.00 & 119 & 53.48 & 4259 & 979807.2 & -2.9 & -148.1 & 0.1 & 1.2 & -148.3 & 4.6 \\
\hline 04LOV315 & 40 & 27.58 & 119 & 53.32 & 4317 & 979804.4 & 0.4 & -146.9 & 0.1 & 1.1 & -147.1 & 5.7 \\
\hline 04LOV316 & 40 & 27.12 & 119 & 53.29 & 4320 & 979804.5 & 1.4 & -145.9 & 0.1 & 1.4 & -145.8 & 6.8 \\
\hline 04LOV317 & 40 & 26.66 & 119 & 53.40 & 4428 & 979796.2 & 4.0 & -147.1 & 0.2 & 1.7 & -146.7 & 5.8 \\
\hline 04LOV318 & 40 & 26.18 & 119 & 53.34 & 4519 & 979792.4 & 9.4 & -144.7 & 0.2 & 1.8 & -144.2 & 8.1 \\
\hline 04LOV319 & 40 & 27.67 & 119 & 45.47 & 3992 & 979809.7 & -25.1 & -161.2 & 0.2 & 0.4 & -162.1 & -9.9 \\
\hline 04LOV320 & 40 & 40.21 & 119 & 42.21 & 5607 & 979734.3 & 32.8 & -158.5 & 0.4 & 4.2 & -155.8 & -0.1 \\
\hline 04LOV321 & 40 & 41.73 & 119 & 42.74 & 5816 & 979722.6 & 38.4 & -160.0 & 0.2 & 2.6 & -158.8 & -2.4 \\
\hline 04LOV322 & 40 & 44.52 & 119 & 42.45 & 6274 & 979692.2 & 46.8 & -167.2 & 0.2 & 2.3 & -166.4 & -8.9 \\
\hline 04LOV323 & 40 & 44.96 & 119 & 39.88 & 6333 & 979689.4 & 48.9 & -167.1 & 0.2 & 2.4 & -166.2 & -8.9 \\
\hline 04LOV324 & 40 & 43.85 & 119 & 41.19 & 6560 & 979673.2 & 55.7 & -168.0 & 0.5 & 4.2 & -165.3 & -8.4 \\
\hline 04LOV325 & 40 & 42.83 & 119 & 37.31 & 5869 & 979715.2 & 34.3 & -165.9 & 0.2 & 3.5 & -163.9 & -7.8 \\
\hline 04LOV326 & 40 & 40.45 & 119 & 39.51 & 5759 & 979724.8 & 37.1 & -159.3 & 0.2 & 4.2 & -156.6 & -1.3 \\
\hline 04LOV327 & 40 & 11.07 & 119 & 41.52 & 3895 & 979796.3 & -22.9 & -155.7 & 0.4 & 1.2 & -155.7 & -4.5 \\
\hline 04LOV328 & 40 & 12.06 & 119 & 40.17 & 3945 & 979797.3 & -18.7 & -153.2 & 0.2 & 1.1 & -153.4 & -2.3 \\
\hline 04LOV329 & 40 & 12.03 & 119 & 38.93 & 3853 & 979800.9 & -23.6 & -155.0 & 0.1 & 1.0 & -155.3 & -4.2 \\
\hline 04LOV330 & 40 & 12.38 & 119 & 37.95 & 3838 & 979799.3 & -27.2 & -158.1 & 0.1 & 1.0 & -158.3 & -7.3 \\
\hline 04LOV331 & 40 & 13.22 & 119 & 37.98 & 3838 & 979800.7 & -27.0 & -157.9 & 0.2 & 1.6 & -157.5 & -6.6 \\
\hline 04LOV332 & 40 & 13.97 & 119 & 37.93 & 3847 & 979802.3 & -25.7 & -156.9 & 0.0 & 1.1 & -157.1 & -6.2 \\
\hline 04LOV333 & 40 & 29.94 & 119 & 57.89 & 5675 & 979714.6 & 34.7 & -158.9 & 0.0 & 1.8 & -158.6 & -5.1 \\
\hline 04LOV334 & 40 & 27.81 & 119 & 56.18 & 5772 & 979711.1 & 43.4 & -153.4 & 0.3 & 3.5 & -151.4 & 1.3 \\
\hline 04LOV335 & 40 & 25.69 & 119 & 55.35 & 6071 & 979694.6 & 58.2 & -148.9 & 0.1 & 4.3 & -146.0 & 5.9 \\
\hline 04LOV336 & 40 & 22.27 & 119 & 55.84 & 5644 & 979722.6 & 51.1 & -141.4 & 0.0 & 1.9 & -140.9 & 10.5 \\
\hline 04LOV337 & 40 & 21.17 & 119 & 50.82 & 4793 & 979766.2 & 16.4 & -147.1 & 0.3 & 2.1 & -146.4 & 4.9 \\
\hline 04LOV338 & 40 & 19.19 & 119 & 50.50 & 4882 & 979754.2 & 15.8 & -150.7 & 0.3 & 2.0 & -150.1 & 0.9 \\
\hline 04LOV339 & 40 & 16.83 & 119 & 50.90 & 5438 & 979713.7 & 31.0 & -154.5 & 1.4 & 7.1 & -148.8 & 2.0 \\
\hline 04LOV340 & 40 & 16.57 & 119 & 54.31 & 5425 & 979721.5 & 37.9 & -147.1 & 1.0 & 4.5 & -144.1 & 6.8 \\
\hline 04LOV341 & 40 & 15.22 & 119 & 53.36 & 5547 & 979709.4 & 39.3 & -149.9 & 1.3 & 6.4 & -144.9 & 6.0 \\
\hline 04LOV342 & 40 & 13.02 & 119 & 51.75 & 5347 & 979711.7 & 26.1 & -156.3 & 1.3 & 6.7 & -151.0 & 0.0 \\
\hline
\end{tabular}




\begin{tabular}{|c|c|c|c|c|c|c|c|c|c|c|c|c|}
\hline 04LOV400 & 40 & 42.64 & 119 & 26.96 & 3918 & 979824.2 & -39.8 & -173.4 & 0.0 & 1.8 & -172.9 & -18.1 \\
\hline 04LOV401 & 40 & 43.15 & 119 & 27.45 & 3989 & 979822.8 & -35.3 & -171.4 & 0.1 & 2.0 & -170.6 & -15.6 \\
\hline 04LOV402 & 40 & 43.71 & 119 & 27.13 & 4132 & 979815.2 & -30.3 & -171.2 & 0.1 & 3.0 & -169.5 & -14.4 \\
\hline 04LOV403 & 40 & 44.29 & 119 & 29.05 & 3977 & 979825.2 & -35.7 & -171.4 & 0.0 & 2.0 & -170.6 & -14.8 \\
\hline 04LOV404 & 40 & 45.11 & 119 & 29.21 & 4032 & 979820.8 & -36.1 & -173.7 & 0.0 & 2.4 & -172.5 & -16.4 \\
\hline 04LOV405 & 40 & 45.57 & 119 & 29.28 & 4090 & 979816.4 & -35.8 & -175.3 & 0.0 & 2.5 & -174.0 & -17.8 \\
\hline 04LOV406 & 40 & 46.45 & 119 & 29.34 & 4130 & 979815.2 & -34.6 & -175.4 & 0.0 & 3.1 & -173.6 & -17.0 \\
\hline 04LOV407 & 40 & 47.75 & 119 & 30.38 & 4256 & 979809.6 & -30.2 & -175.4 & 0.1 & 2.7 & -174.0 & -16.7 \\
\hline 04LOV408 & 40 & 48.21 & 119 & 29.34 & 4557 & 979791.5 & -20.7 & -176.2 & 0.1 & 3.9 & -173.6 & -16.3 \\
\hline 04LOV409 & 40 & 48.82 & 119 & 29.76 & 4683 & 979781.0 & -20.3 & -180.0 & 0.1 & 3.5 & -177.9 & -20.3 \\
\hline 04LOV410 & 40 & 49.91 & 119 & 30.15 & 4984 & 979770.6 & -4.1 & -174.0 & 0.2 & 4.2 & -171.2 & -13.3 \\
\hline 04LOV411 & 40 & 49.99 & 119 & 29.62 & 5470 & 979740.9 & 11.8 & -174.7 & 1.5 & 6.3 & -169.9 & -12.1 \\
\hline 04LOV412 & 40 & 50.38 & 119 & 30.78 & 4941 & 979775.7 & -3.6 & -172.1 & & 3.5 & -170.0 & -11.6 \\
\hline 04LOV413 & 40 & 52.32 & 119 & 36.08 & 5707 & 979731.0 & 20.8 & -173.9 & 0.2 & 1.4 & -174.0 & -14.0 \\
\hline 04LOV414 & 40 & 51.66 & 119 & 36.34 & 5545 & 979745.0 & 20.5 & -168.7 & 0.7 & 1.9 & -168.2 & -8.3 \\
\hline 04LOV415 & 40 & 51.22 & 119 & 34.28 & 4895 & 979786.9 & 2.0 & -165.0 & 0.8 & 2.6 & -163.8 & -4.4 \\
\hline 04LOV416 & 40 & 50.64 & 119 & 33.65 & 4585 & 979800.7 & -12.5 & -168.9 & 0.1 & 2.1 & -168.2 & -9.0 \\
\hline 04LOV417 & 40 & 48.35 & 119 & 30.97 & 4282 & 979809.9 & -28.4 & -174.4 & 0.1 & 2.7 & -173.0 & -15.3 \\
\hline 04LOV418 & 40 & 40.91 & 119 & 35.13 & 3849 & 979841.3 & -26.6 & -157.9 & 0.0 & 1.6 & -157.5 & -2.0 \\
\hline 04LOV419 & 40 & 40.79 & 119 & 34.93 & 3847 & 979840.7 & -27.3 & -158.5 & 0.0 & 1.4 & -158.3 & -3.0 \\
\hline 04LOV420 & 40 & 40.65 & 119 & 34.70 & 3849 & 39.5 & -28.1 & -159.3 & 0.0 & 1.1 & -159.4 & -4.2 \\
\hline 04LOV421 & 40 & 40.51 & 119 & 34.49 & 3848 & 979838.3 & -29.1 & -160.4 & 0.0 & 1.0 & -160.6 & -5.4 \\
\hline 04LOV422 & 40 & 40.37 & 119 & 34.27 & 3851 & 979836.8 & -30.1 & -161.4 & 0.0 & 0.9 & -161.8 & -6.8 \\
\hline 04LOV423 & 40 & 40.24 & 119 & 34.06 & 3849 & 979834.7 & -32.2 & -163.4 & 0.0 & 0.8 & -163.9 & -8.9 \\
\hline 04LOV424 & 40 & 40.10 & 119 & 33.84 & 3849 & 979832.9 & -33.8 & -165.1 & 0.0 & 0.7 & -165.6 & -10.7 \\
\hline 04LOV425 & 40 & 39.97 & 119 & 33.63 & 3849 & 31.8 & -34.7 & -166.0 & 0.0 & 0.6 & -166.6 & -11.8 \\
\hline 04LOV426 & 40 & 39.83 & 119 & 33.41 & 3851 & 979831.3 & -34.8 & -166.2 & 0.0 & 0.5 & -166.8 & -12.1 \\
\hline 04LOV427 & 40 & 39.68 & 119 & 33.21 & 3850 & 979831.3 & -34.7 & -166.0 & 0.0 & 0.5 & -166.7 & -12.1 \\
\hline 04LOV428 & 40 & 39.54 & 119 & 32.98 & 3850 & 979831.5 & -34.3 & -165.6 & 0.0 & 0.5 & -166.4 & -11.8 \\
\hline 04LOV429 & 40 & 39.41 & 119 & 32.77 & 3850 & 979831.7 & -33.9 & -165.2 & & 0.4 & -166.0 & -11.5 \\
\hline 04LOV430 & 40 & 39.29 & 119 & 32.53 & 3851 & 979831.5 & -33.9 & -165.2 & 0.0 & 0.4 & -166.0 & -11.6 \\
\hline 04LOV431 & 40 & 39.14 & 119 & 32.33 & 3852 & 979830.7 & -34.3 & -165.7 & 0.0 & 0.4 & -166.6 & -12.2 \\
\hline 04LOV432 & 40 & 38.99 & 119 & 32.11 & 3850 & 979829.5 & -35.5 & -166.8 & 0.0 & 0.3 & -167.7 & -13.4 \\
\hline 04LOV433 & 40 & 38.87 & 119 & 31.90 & 3850 & 979828.6 & -36.1 & -167.4 & 0.0 & 0.3 & -168.3 & -14.1 \\
\hline 04LOV434 & 40 & 38.73 & 119 & 31.67 & 3848 & 979828.4 & -36.4 & -167.6 & 0.0 & 0.3 & -168.5 & -14.4 \\
\hline 04LOV435 & 40 & 38.60 & 119 & 31.46 & 3848 & 979828.0 & -36.6 & -167.9 & 0.0 & 0.3 & -168.8 & -14.7 \\
\hline 04LOV436 & 40 & 38.45 & 119 & 31.24 & 3849 & 979827.0 & -37.2 & -168.5 & 0.0 & 0.3 & -169.4 & -15.4 \\
\hline 04LOV437 & 40 & 38.31 & 119 & 31.05 & 3851 & 979826.1 & -37.7 & -169.1 & 0.0 & 0.3 & -170.0 & -16.1 \\
\hline 04LOV438 & 40 & 38.19 & 119 & 30.82 & 3858 & 979825.3 & -37.7 & -169.3 & 0.0 & 0.3 & -170.2 & -16.4 \\
\hline 04LOV439 & 40 & 38.03 & 119 & 30.53 & 3860 & 979825.7 & -36.9 & -168.6 & 0.0 & 0.3 & -169.5 & -15.7 \\
\hline 04LOV440 & 40 & 38.01 & 119 & 30.78 & 3855 & 979825.0 & -38.0 & -16 & 0.0 & 0.3 & -170.4 & -16.6 \\
\hline 04LOV441 & 40 & 38.01 & 119 & 31.04 & 3852 & 979825.3 & -38.0 & -169.4 & 0.0 & 0.3 & -170.3 & -16.4 \\
\hline 04LOV442 & 40 & 38.00 & 119 & 31.32 & 3850 & 979826.0 & -37.5 & -168.8 & 0.0 & 0.3 & -169.7 & -15.9 \\
\hline 04LOV443 & 40 & 38.01 & 119 & 31.61 & 3850 & 979826.8 & -36.8 & -168.0 & 0.0 & 0.3 & -169.0 & -15.1 \\
\hline 04LOV444 & 40 & 38.01 & 119 & 31.89 & 3850 & 979827.5 & -36.0 & -167.3 & 0.0 & 0.3 & -168.2 & -14.3 \\
\hline 04LOV445 & 40 & 38.01 & 119 & 32.17 & 3851 & 979828.0 & -35.4 & -166.8 & 0.0 & 0.3 & -167.7 & -13.7 \\
\hline 04LOV446 & 40 & 38.01 & 119 & 32.44 & 3851 & 979828.1 & -35.3 & -166.7 & 0.0 & 0.3 & -167.6 & -13.6 \\
\hline 04LOV447 & 40 & 38.01 & 119 & 32.73 & 3851 & 979827.7 & -35.7 & -167.1 & 0.0 & 0.3 & -168.0 & -14.0 \\
\hline 04LOV448 & 40 & 38.01 & 119 & 33.01 & 3851 & 979827.5 & -35.9 & -167.2 & 0.0 & 0.3 & -168.2 & -14.2 \\
\hline 04LOV449 & 40 & 38.01 & 119 & 33.37 & 3849 & 979827.1 & -36.5 & -167.8 & 0.0 & 0.3 & -168.7 & -14.6 \\
\hline
\end{tabular}




\begin{tabular}{|c|c|c|c|c|c|c|c|c|c|c|c|c|}
\hline 04LOV450 & 40 & 38.01 & 119 & 33.75 & 3849 & 979827.2 & -36.4 & -167.7 & 0.0 & 0.3 & -168.6 & -14.4 \\
\hline 04LOV451 & 40 & 38.01 & 119 & 34.04 & 3849 & 979827.2 & -36.3 & -167.6 & 0.0 & 0.3 & -168.5 & -14.3 \\
\hline 04LOV452 & 40 & 38.01 & 119 & 34.33 & 3848 & 979827.5 & -36.2 & -167.5 & 0.0 & 0.4 & -168.3 & -14.1 \\
\hline 04LOV453 & 40 & 38.01 & 119 & 34.62 & 3847 & 979828.1 & -35.7 & -166.9 & 0.0 & 0.4 & -167.7 & -13.5 \\
\hline 04LOV454 & 40 & 38.02 & 119 & 34.90 & 3847 & 979829.2 & -34.6 & -165.8 & 0.0 & 0.4 & -166.6 & -12.3 \\
\hline 04LOV455 & 40 & 38.01 & 119 & 35.19 & 3848 & 979831.1 & -32.5 & -163.8 & 0.0 & 0.4 & -164.6 & -10.2 \\
\hline 04LOV456 & 40 & 38.01 & 119 & 35.48 & 3849 & 979833.1 & -30.5 & -161.7 & 0.0 & 0.5 & -162.5 & -8.2 \\
\hline 04LOV457 & 40 & 38.02 & 119 & 35.77 & 3852 & 979834.9 & -28.4 & -159.8 & 0.0 & 0.5 & -160.5 & -6.2 \\
\hline 04LOV458 & 40 & 38.01 & 119 & 36.05 & 3848 & 979837.0 & -26.7 & -157.9 & 0.0 & 0.5 & -158.6 & -4.2 \\
\hline 04LOV459 & 40 & 38.01 & 119 & 36.34 & 3846 & 979839.4 & -24.5 & -155.6 & 0.0 & 0.6 & -156.3 & -1.9 \\
\hline 04LOV460 & 40 & 38.01 & 119 & 36.61 & 3845 & 979841.6 & -22.4 & -153.6 & 0.0 & 0.6 & -154.2 & 0.3 \\
\hline 04LOV461 & 40 & 37.85 & 119 & 36.24 & 3845 & 979837.9 & -25.9 & -157.0 & 0.0 & 0.5 & -157.7 & -3.4 \\
\hline 04LOV462 & 40 & 37.71 & 119 & 36.04 & 3847 & 979835.8 & -27.5 & -158.8 & 0.0 & 0.5 & -159.5 & -5.3 \\
\hline 04LOV463 & 40 & 37.56 & 119 & 35.82 & 3849 & 979833.5 & -29.4 & -160.7 & 0.0 & 0.4 & -161.5 & -7.3 \\
\hline 04LOV464 & 40 & 37.42 & 119 & 35.60 & 3848 & 979831.1 & -31.7 & -162.9 & 0.0 & 0.4 & -163.8 & -9.7 \\
\hline 04LOV465 & 40 & 37.28 & 119 & 35.39 & 3848 & 979829.1 & -33.5 & -164.8 & 0.0 & 0.4 & -165.6 & -11.6 \\
\hline 04LOV466 & 40 & 37.13 & 119 & 35.18 & 3849 & 979827.6 & -34.6 & -165.9 & 0.0 & 0.3 & -166.8 & -12.8 \\
\hline 04LOV467 & 40 & 36.99 & 119 & 34.96 & 3849 & 979826.9 & -35.1 & -166.4 & 0.0 & 0.3 & -167.3 & -13.4 \\
\hline 04LOV468 & 40 & 36.84 & 119 & 34.75 & 3847 & 979826.6 & -35.5 & -166.7 & 0.0 & 0.3 & -167.6 & -13.7 \\
\hline 04LOV469 & 40 & 36.70 & 119 & 34.55 & 3846 & 979826.2 & -35.7 & -166.9 & 0.0 & 0.3 & -167.8 & -14.0 \\
\hline 04LOV470 & 40 & 36.57 & 119 & 34.34 & 3847 & 979825.8 & -35.8 & -167.0 & 0.0 & 0.3 & -167.9 & -14.2 \\
\hline 04LOV471 & 40 & 36.42 & 119 & 34.10 & 3846 & 979825.3 & -36.2 & -167.4 & 0.0 & 0.3 & -168.3 & -14.6 \\
\hline 04LOV472 & 40 & 36.28 & 119 & 33.89 & 3846 & 979825.1 & -36.3 & -167.4 & 0.0 & 0.3 & -168.3 & -14.7 \\
\hline 04LOV473 & 40 & 36.14 & 119 & 33.67 & 3846 & 979825.0 & -36.1 & -167.3 & 0.0 & 0.4 & -168.1 & -14.6 \\
\hline 04LOV474 & 40 & 35.98 & 119 & 33.45 & 3845 & 979825.2 & -35.8 & -166.9 & 0.0 & 0.4 & -167.7 & -14.3 \\
\hline 04LOV475 & 40 & 35.85 & 119 & 33.22 & 3844 & 979825.4 & -35.4 & -166.5 & 0.0 & 0.5 & -167.3 & -13.9 \\
\hline 04LOV476 & 40 & 36.46 & 119 & 35.10 & 3844 & 979826.4 & -35.3 & -166.4 & 0.0 & 0.3 & -167.3 & -13.5 \\
\hline 04LOV477 & 40 & 36.51 & 119 & 36.30 & 3848 & 979831.4 & -30.0 & -161.3 & 0.0 & 0.3 & -162.2 & -8.3 \\
\hline 04LOV478 & 40 & 36.50 & 119 & 37.43 & 3841 & 979834.1 & -28.0 & -159.0 & 0.0 & 0.4 & -159.8 & -5.8 \\
\hline 04LOV500 & 40 & 14.67 & 119 & 41.64 & 5005 & 979735.9 & 15.8 & -154.9 & 0.4 & 2.9 & -153.5 & -2.8 \\
\hline 04LOV501 & 40 & 16.51 & 119 & 40.31 & 4977 & 979737.9 & 12.4 & -157.4 & 1.3 & 5.3 & -153.5 & -2.9 \\
\hline 04LOV502 & 40 & 18.48 & 119 & 41.81 & 4671 & 979761.8 & 4.6 & -154.7 & 0.6 & 2.7 & -153.4 & -2.7 \\
\hline 04LOV503 & 40 & 21.82 & 119 & 36.61 & 6456 & 979667.6 & 73.2 & -147.0 & 0.5 & 6.9 & -141.7 & 8.8 \\
\hline 04LOV504 & 40 & 23.36 & 119 & 36.28 & 7095 & 979623.9 & 87.2 & -154.8 & 0.2 & 10.5 & -145.8 & 4.7 \\
\hline 04LOV505 & 40 & 26.03 & 119 & 34.18 & 6890 & 979645.1 & 85.2 & -149.8 & 1.0 & 11.7 & -139.6 & 11.2 \\
\hline 04LOV506 & 40 & 30.40 & 119 & 32.66 & 5971 & 979703.4 & 50.6 & -153.0 & 0.5 & 4.7 & -149.8 & 1.8 \\
\hline 04LOV507 & 40 & 30.03 & 119 & 34.86 & 6498 & 979672.9 & 70.3 & -151.4 & 0.7 & 10.2 & -142.7 & 8.8 \\
\hline 05LOV001 & 40 & 13.84 & 119 & 44.99 & 4937 & 979742.5 & 17.2 & -151.2 & 0.6 & 1.7 & -151.0 & -0.2 \\
\hline 05LOV002 & 40 & 12.89 & 119 & 42.62 & 5122 & 979724.0 & 17.4 & -157.2 & 2.3 & 4.0 & -154.7 & -3.9 \\
\hline 05LOV003 & 40 & 14.17 & 119 & 43.27 & 5293 & 979715.2 & 22.9 & -157.7 & 1.5 & 3.8 & -155.3 & -4.6 \\
\hline 05LOV004 & 40 & 15.36 & 119 & 40.01 & 4683 & 979759.2 & 7.7 & -152.0 & 0.6 & 1.3 & -152.0 & -1.3 \\
\hline 05LOV005 & 40 & 20.52 & 119 & 37.66 & 6357 & 979665.1 & 63.4 & -153.5 & 6.4 & 13.8 & -141.2 & 9.2 \\
\hline 05LOV006 & 40 & 23.02 & 119 & 37.94 & 6282 & 979677.2 & 64.6 & -149.7 & 3.2 & 9.1 & -142.1 & 8.5 \\
\hline 05LOV007 & 40 & 22.34 & 119 & 33.56 & 6305 & 979679.5 & 70.1 & -145.0 & 1.6 & 5.7 & -140.8 & 9.8 \\
\hline 05LOV008 & 40 & 25.29 & 119 & 35.18 & 6404 & 979673.5 & 69.1 & -149.4 & 3.1 & 8.1 & -142.8 & 8.0 \\
\hline 05LOV009 & 40 & 24.71 & 119 & 37.84 & 6021 & 979691.3 & 51.6 & -153.7 & 4.2 & 9.5 & -145.7 & 5.2 \\
\hline 05LOV010 & 40 & 26.76 & 119 & 36.66 & 4824 & 979774.5 & 19.3 & -145.3 & 2.6 & 4.5 & -142.1 & 9.3 \\
\hline
\end{tabular}




\begin{tabular}{|c|c|c|c|c|c|c|c|c|c|c|c|c|}
\hline 05LOV011 & 40 & 27.21 & 119 & 34.55 & 6186 & 979692.3 & 64.5 & -146.5 & 2.2 & 6.5 & -141.5 & 9.6 \\
\hline 05LOV012 & 40 & 27.87 & 119 & 35.44 & 5768 & 979718.2 & 50.1 & -146.6 & 1.6 & 5.1 & -143.0 & 8.3 \\
\hline LOV013 & 40 & 28.99 & 119 & 36.78 & 4319 & 979803.4 & -2.6 & -149.9 & 2.3 & 4.3 & -146.9 & 50 \\
\hline 5LOV014 & 40 & 29.86 & 119 & 36.06 & 959 & 979698.7 & 45.6 & -157.7 & 8.4 & 14.4 & -144.8 & \\
\hline 5LOV015 & 40 & 1.56 & 119 & 34.23 & 000 & 979699.1 & 47.3 & -157.4 & 5.8 & 11.5 & -147.4 & \\
\hline 5LOV016 & 40 & 2.93 & 119 & 32.99 & 497 & 979736.2 & 35.0 & -152.5 & 4.2 & 7.5 & -146.4 & \\
\hline LOV017 & 40 & 2.85 & 119 & 30.93 & 5068 & 979763.1 & 21.8 & -151.1 & 1.8 & 3.1 & -149.4 & \\
\hline 5LOV018 & 40 & 4.07 & 119 & 31.16 & 434 & 979742.3 & 33.6 & -151.7 & 4.0 & 7.3 & -145.9 & \\
\hline 5LOV019 & 40 & 5.13 & 119 & 30.36 & 664 & 979793.0 & 10.3 & -148.8 & 1.5 & 2.4 & & \\
\hline 5LOV020 & 40 & 36.00 & 119 & 30.89 & 3859 & 979830.2 & -29.5 & -161.1 & 0.2 & 1.0 & & -8.0 \\
\hline $5 \mathrm{LOV} 021$ & 40 & 36.75 & 119 & 29.13 & 367 & 979813.5 & 0.5 & -148.4 & 2.2 & 2.6 & & \\
\hline 5LOV022 & 40 & 7.12 & 119 & 27.91 & 394 & 979810.0 & -1.0 & -150.9 & 1.8 & 2.3 & 49.9 & \\
\hline 5LOV023 & 40 & 5.96 & 119 & 28.35 & 4777 & 979786.0 & 12.6 & -150.3 & 4.3 & 5.6 & -146.1 & \\
\hline 05LOV024 & 40 & 3.88 & 119 & 29.46 & 5083 & 979764.8 & 23.4 & -150.0 & 3.5 & 5.4 & & \\
\hline 05LOV025 & 40 & 31.35 & 119 & 30.87 & 5229 & 97975 & 28.2 & -150.2 & 1.6 & 3.2 & & \\
\hline $5 \mathrm{LOV} 026$ & 40 & 1.81 & 119 & 32.14 & 126 & 979758.6 & 24.3 & -150.5 & 0.9 & 2.3 & & \\
\hline 5LOV027 & 40 & 29.13 & 119 & 32.87 & 173 & 979693.6 & 61.7 & -148.8 & 1.8 & 5.9 & 44.5 & \\
\hline $5 \mathrm{LOV} 028$ & 40 & 49.47 & 119 & 34.65 & 244 & 979761.2 & 11.7 & -167.2 & 0.5 & 1.6 & -167.1 & -8.4 \\
\hline 029 & 40 & 9.92 & 119 & 36.98 & 045 & 979711.8 & 36.9 & -169.3 & 1. & 3.2 & & -8.5 \\
\hline $5 \mathrm{LO}$ & 40 & 8.02 & 119 & 37.81 & 292 & 9796 & 43.6 & -171.0 & 0 . & 2.6 & & -11.5 \\
\hline $5 \mathrm{LOV} 031$ & 40 & 47.40 & 119 & 35.76 & 5777 & 979721.6 & 25.2 & -171.8 & 1.0 & 2.6 & & -12.9 \\
\hline $5 \mathrm{LOV} 032$ & 40 & 48.30 & 119 & 33.61 & 5278 & 979748.2 & 3.6 & -176.4 & 1.8 & 3.1 & 74.8 & -16.8 \\
\hline J033 & 40 & 47.22 & 119 & 31.90 & 4718 & 979780.2 & -15.4 & -176.3 & 0.9 & 2.2 & -175.5 & -18.2 \\
\hline 034 & 40 & 45.59 & 119 & 31.02 & 4610 & 979786.7 & -16.6 & -173.8 & 1. & 2.4 & 72.8 & -16.3 \\
\hline $05 \mathrm{LC}$ & 40 & 45.55 & 119 & 33.12 & 4858 & $979^{\circ}$ & -5.1 & -170.8 & 1.0 & 2.4 & & -13.1 \\
\hline $05 \mathrm{LC}$ & 40 & 45.96 & 119 & 36.84 & 6116 & & 38.1 & -170.5 & & 3.0 & & -11.8 \\
\hline V037 & 40 & 44.84 & 119 & 37.25 & 994 & 979708.1 & 36.0 & -168.5 & 0. & 2.3 & & -10.8 \\
\hline 5LOV038 & 40 & 43.86 & 119 & 35.63 & 558 & 979731.7 & 20.0 & -169.6 & 2. & 4.5 & -166.5 & -10.2 \\
\hline 05LOV039 & 40 & 43.58 & 119 & 38.69 & 874 & 979716.0 & 34.5 & -165.9 & 0.6 & 2.4 & 65.0 & -8.5 \\
\hline 05LC & 40 & 1.73 & 119 & 37.73 & 671 & 979 & 30.2 & -163.2 & 1. & 4.3 & & -4.7 \\
\hline $05 \mathrm{LO}$ & 40 & 42.15 & 119 & 39.92 & 983 & 979711.6 & 42.4 & -161.7 & 0.8 & 3.1 & & -4.0 \\
\hline V042 & 40 & 42.89 & 119 & 45.41 & 894 & & 33.7 & -167.3 & 1.5 & 3.7 & & -7.9 \\
\hline 5LOV043 & 40 & 38.39 & 119 & 40.30 & 4592 & 979797.0 & 2.7 & -153.9 & 0.4 & 1.2 & 4.0 & 0.9 \\
\hline J044 & 40 & 39.13 & 119 & 43.71 & 133 & 979816.3 & -22.2 & -163.2 & 0. & 1.6 & 62.9 & $-7 .($ \\
\hline 045 & 40 & 40.40 & 119 & 44.21 & 4511 & 979797.6 & -7.3 & -161.1 & 1. & 2.7 & -159.8 & -3.5 \\
\hline 05LOV046 & 40 & 39.02 & 119 & 48.23 & 4483 & 979791.1 & -14.5 & -167.4 & 0.8 & 1.3 & -167.4 & -11.1 \\
\hline 05LOV047 & 40 & 37.43 & 119 & 55.08 & 5283 & 979747.0 & 19.1 & -161.1 & 1.0 & 1.9 & & -4.4 \\
\hline 05LOV048 & 40 & 36.51 & 119 & 52.45 & 5665 & 979716.6 & 25.9 & -167.3 & 1.8 & 4.1 & & -9.2 \\
\hline 05LOV049 & 40 & 35.43 & 119 & 50.58 & 4795 & 979769.1 & -1.7 & -165.2 & 0. & 1.6 & -165.0 & -10.0 \\
\hline 05LOV050 & 40 & 33.51 & 119 & 50.70 & 4737 & 979772.6 & -0.8 & -162.3 & 1. & 1.7 & -162.0 & -7.8 \\
\hline $05 \mathrm{LO}$ & 40 & 36.04 & 119 & 48.10 & 4137 & 979808.4 & -25.1 & -166.2 & 0.2 & 0.6 & -166.9 & -11.8 \\
\hline 05LOV052 & 40 & 32.35 & 119 & 46.22 & 3864 & 979813.8 & -40.0 & -171.7 & 0.0 & 0.2 & -172.8 & -19.2 \\
\hline 05LOV053 & 40 & 31.07 & 119 & 48.08 & 3881 & 979813.5 & -36.8 & -169.1 & 0.0 & 0.2 & -170.2 & -16.8 \\
\hline 05LOV054 & 40 & 31.03 & 119 & 46.13 & 3854 & 979812.7 & -40.0 & -171.5 & 0.0 & 0.1 & -172.6 & -19.4 \\
\hline $05 \mathrm{LO}$ & 40 & 31.10 & 119 & 44.44 & 878 & 979812.7 & -37.8 & -170.1 & 0.0 & 0.1 & -171.2 & -18 \\
\hline 05LOV056 & 40 & 31.06 & 119 & 42.68 & 3849 & 979812.0 & -41.3 & -172.6 & 0.0 & 0.1 & -173.7 & -20 \\
\hline 05LOV057 & 40 & 32.38 & 119 & 44.18 & 3892 & 979815.0 & -36.2 & -169.0 & 0.0 & 0.1 & -170.1 & -16.7 \\
\hline 05LOV058 & 40 & 27.34 & 119 & 43.94 & 3845 & 979809.8 & -38.3 & -169.4 & 0.0 & 0.1 & -170.5 & -18.5 \\
\hline 05LOV059 & 40 & 28.29 & 119 & 43.95 & 3838 & 979810.4 & -39.7 & -170.6 & 0.0 & 0.1 & -171.7 & -19 \\
\hline 55LOV060 & 40 & 29.16 & 119 & 43.95 & 3842 & 979809.5 & -41.6 & -172.6 & 0.0 & 0.1 & -173.8 & -21 \\
\hline
\end{tabular}




\begin{tabular}{|c|c|c|c|c|c|c|c|c|c|c|c|c|}
\hline 05LOV061 & 40 & 29.14 & 119 & 45.36 & 3861 & 979815.3 & -33.9 & -165.6 & 0.0 & 0.1 & -166.7 & -14.2 \\
\hline 05LOV062 & 40 & 28.21 & 119 & 45.39 & 3871 & 979816.8 & -30.1 & -162.1 & 0.0 & 0.1 & -163.2 & -10.9 \\
\hline 5LOV063 & 40 & 27.15 & 119 & 45.34 & 3852 & 979812.1 & -35.0 & -166.4 & 0.0 & 0.1 & -167.5 & -15.4 \\
\hline 05LOV064 & 40 & 29.17 & 119 & 46.75 & 3845 & 979814.7 & -36.1 & -167.2 & 0.0 & 0.1 & -168.3 & -15.7 \\
\hline 05LOV065 & 40 & 28.20 & 119 & 46.70 & 3846 & 979814.7 & -34.5 & -165.7 & 0.0 & .1 & -166.8 & \\
\hline $5 \mathrm{LOV} 066$ & 40 & 27.30 & 119 & 46.64 & 843 & 979810.6 & -37.7 & -168.7 & 0.0 & 0.1 & -169.9 & -17. \\
\hline $5 \mathrm{LOV} 067$ & 40 & 27.26 & 119 & 48.27 & 854 & 979805.2 & -41.9 & -173.4 & 0.0 & 0.2 & -174.4 & \\
\hline 05LOV068 & 40 & 28.22 & 119 & 48.21 & 864 & 979811.2 & -36.4 & -168.2 & 0.0 & .2 & -169.3 & -16 \\
\hline 05LOV069 & 40 & 29.20 & 119 & 48.23 & 851 & 979815.4 & -34.8 & -166.2 & 0.0 & .2 & -167.2 & -14 \\
\hline 5LOV070 & 40 & 28.51 & 119 & 45.77 & 956 & & -24.6 & -159.5 & 0.2 & .2 & 60.6 & \\
\hline 05LOV071 & 40 & 20.65 & 119 & 39.41 & 295 & 979792.9 & -2.9 & -149.4 & 0.4 & 1.9 & -148.8 & \\
\hline 05LOV072 & 40 & 21.73 & 119 & 38.13 & 015 & 979760.7 & 30.9 & -140.1 & 6.0 & 8.1 & -133.4 & \\
\hline 05LOV073 & 40 & 21.45 & 119 & 38.98 & 4622 & 97977 & 12.7 & -145.0 & & .0 & & \\
\hline 05LOV074 & 40 & 41.37 & 119 & 35.72 & 3950 & 979 & -23.5 & -158.2 & 0.9 & 3.1 & -156.3 & \\
\hline 05LOV075 & 40 & 41.90 & 119 & 35.95 & 350 & & -11.9 & -160.2 & 2.6 & 4.4 & 57.2 & \\
\hline 05LOV076 & 40 & 40.32 & 119 & 37.45 & 4049 & & -13.9 & -152.0 & 2.5 & 4.6 & -148.7 & \\
\hline 5LOV077 & 40 & 42.99 & 119 & 25.48 & 4152 & & -29.7 & -171.3 & 0.7 & 3.9 & -168.7 & \\
\hline 05LOV078 & 40 & 44.52 & 119 & 26.21 & 4787 & $979^{\prime}$ & -9.8 & -173.1 & 5.0 & 9.4 & -165.0 & \\
\hline 05LOV079 & 40 & 37.32 & 119 & 44.50 & 3934 & 979 & -32.8 & -167.0 & 0.0 & 0.5 & -167.7 & -12 \\
\hline $05 \mathrm{LO}$ & 40 & 38.50 & 119 & 46.23 & 989 & & -29.9 & -166.0 & & .0 & 66.2 & -10. \\
\hline 081 & 40 & 37.91 & 119 & 46.28 & 967 & & -32.9 & -168.2 & 0 . & .7 & 68.7 & -13 \\
\hline V082 & 40 & 35.93 & 119 & 43.96 & 3883 & & -35.8 & -168.2 & 0.0 & 0.3 & -169.1 & -14. \\
\hline $05 \mathrm{LO}$ & 40 & 35.56 & 119 & 43.83 & 876 & & -35.8 & -168.0 & 0.0 & .3 & -169.0 & -14 \\
\hline 05LOV084 & 40 & 35.16 & 119 & 43.72 & 871 & 979 & -35.9 & -167.9 & 0. & .2 & -168.9 & -14 \\
\hline 05LOV085 & 40 & 34.84 & 119 & 43.83 & 3866 & 979 & -35.4 & -167.2 & 0.0 & 0.2 & -168.3 & -14 . \\
\hline $05 \mathrm{LO}$ & 40 & 34.43 & 119 & 43.54 & 3872 & & -34.4 & -166.4 & & .2 & -167.5 & -13 \\
\hline 087 & 40 & 34.64 & 119 & 42.67 & & & -36.2 & -167.5 & & .2 & -168.6 & -14 \\
\hline V088 & 40 & 35.09 & 119 & 42.70 & 3861 & & -36.4 & -168.1 & 0.0 & .2 & -169.1 & -15. \\
\hline 05LOV089 & 40 & 35.76 & 119 & 42.75 & 3883 & 979 & -34.7 & -167.1 & & 0.3 & -168.1 & -13 . \\
\hline $05 \mathrm{LO}$ & 40 & 17.34 & 119 & 48.16 & 3930 & & -13.5 & -147.5 & & & 48.1 & \\
\hline $5 \mathrm{LO}$ & 40 & 17.01 & 119 & 48.37 & 4073 & & -7.3 & -146.2 & 0 & 0.9 & -146.6 & \\
\hline $05 \mathrm{LO}$ & 40 & 19.03 & 119 & 48.20 & 3898 & & -24.4 & -157.4 & 0.0 & .4 & -158.2 & \\
\hline 05LOV093 & 40 & 18.97 & 119 & 48.73 & 3935 & & -17.6 & -151.9 & 0.2 & 0.7 & -152.4 & \\
\hline 5LOV094 & 40 & 19.14 & 119 & 49.33 & 4185 & & -5.7 & -148.4 & 1.1 & 1.4 & -148.3 & \\
\hline 05LOV095 & 40 & 24.17 & 119 & 50.20 & 3933 & & -14.0 & -148.2 & & 0.8 & -148.7 & \\
\hline $05 \mathrm{LO}$ & 40 & 24.02 & 119 & 50.95 & 4129 & 979 & -1.9 & -142.7 & 0.5 & 1.0 & -143.0 & \\
\hline 05LOV097 & 40 & 23.90 & 119 & 51.61 & 4274 & 979 & 4.8 & -141.0 & 0.2 & 0.9 & -141.4 & \\
\hline 05LOV098 & 40 & 25.07 & 119 & 50.89 & 3958 & & -15.3 & -150.3 & 0.2 & 0.9 & -150.6 & \\
\hline 05LOV099 & 40 & 25.63 & 119 & 51.03 & 3938 & 979816.9 & -19.8 & -154.1 & 0.1 & 0.9 & -154.5 & -2 . \\
\hline 05LOV100 & 40 & 25.64 & 119 & 51.74 & 4155 & 979811.1 & -5.2 & -147.0 & & 1.6 & -146.7 & \\
\hline $05 \mathrm{LO}$ & 40 & 30.06 & & 50.29 & & & & -156.4 & & & 57.3 & -4. \\
\hline 05LOV102 & 40 & 30.14 & 119 & 49.64 & 3931 & 979822.3 & -21.9 & -156.0 & 0.0 & 0.3 & -156.9 & \\
\hline 05LOV103 & 40 & 30.71 & 119 & 49.20 & 3944 & 979819.0 & -24.7 & -159.3 & 0.0 & 0.3 & -160.2 & -6 \\
\hline 05LOV104 & 40 & 31.06 & 119 & 49.20 & 3939 & 979817.6 & -27.2 & -161.6 & 0.1 & 0.3 & -162.5 & -9 \\
\hline 05LOV500 & 40 & 39.39 & 119 & 37.81 & 4001 & 979834.2 & -17.1 & -153.6 & 2.2 & 3.7 & -151.2 & \\
\hline 05LOV501 & 40 & 37.29 & 119 & 38.84 & 4083 & & -10.4 & -149.6 & 0. & 1.0 & -149.9 & \\
\hline 05LOV502 & 40 & 37.86 & 119 & 38.22 & 3971 & & -10.0 & -145.4 & 0.7 & 1.3 & & \\
\hline 05LOV503 & 40 & 37.74 & 119 & 38.57 & 4008 & 979835.9 & -12.4 & -149.1 & 0.9 & 1.5 & -148.8 & \\
\hline 05LOV504 & 40 & 43.41 & 119 & 33.01 & 3902 & 979834.7 & -31.9 & -165.0 & 0.2 & 1.9 & -164.3 & \\
\hline 05LOV505 & 40 & 43.62 & 119 & 33.83 & 3980 & 979830.3 & -29.4 & -165.1 & 0.9 & 3.3 & -163.1 & \\
\hline
\end{tabular}




\begin{tabular}{|c|c|c|c|c|c|c|c|c|c|c|c|c|}
\hline 05LOV506 & 40 & 43.42 & 119 & 34.01 & 3954 & 979832.9 & -28.8 & -163.7 & 0.7 & 3.2 & -161.7 & -5.5 \\
\hline 05LOV507 & 40 & 43.89 & 119 & 35.02 & 4783 & 979780.3 & -4.2 & -167.4 & 3.5 & 5.3 & -163.5 & -7.1 \\
\hline 5LOV508 & 40 & 43.75 & 119 & 34.94 & 4572 & 979793.9 & -10.3 & -166.2 & 3.3 & .2 & -162.3 & -5.9 \\
\hline 5LOV509 & 40 & 43.66 & 119 & 34.71 & 4304 & 979810.7 & -18.6 & -165.4 & 2.9 & 5.2 & -161.5 & -5.1 \\
\hline 5LOV510 & 40 & 43.44 & 119 & 34.53 & 4088 & 979824.4 & -24.8 & -164.3 & 1.9 & 4.5 & -161.0 & -4.7 \\
\hline 5LOV511 & 40 & 43.48 & 119 & 34.28 & 4002 & 979829.7 & -27.7 & -164.2 & 1.5 & 4.3 & -161.2 & -4.8 \\
\hline 5LOV512 & 40 & 43.64 & 119 & 33.24 & 3867 & 979835.7 & -34.6 & -166.5 & 0.4 & 2.6 & -165.1 & -8.9 \\
\hline 5LOV513 & 40 & 43.81 & 119 & 32.18 & 3874 & 979833.1 & -36.8 & -168.9 & 0.1 & 1.7 & -168.4 & -12.3 \\
\hline 5LOV514 & 40 & 43.81 & 119 & 31.82 & 3897 & 979831.9 & -35.8 & -168.7 & 0.1 & 1.6 & -168.4 & -12.4 \\
\hline $5 \mathrm{LOV} 515$ & 40 & 39.54 & 119 & 37.91 & 4185 & 979823.7 & -10.5 & -153.3 & 2.9 & 4.2 & -150.4 & 4.8 \\
\hline $5 \mathrm{LOV} 516$ & 40 & 39.40 & 119 & 37.96 & 4289 & 979817.8 & -6.5 & -152.8 & 2.4 & 3.5 & -150.6 & 4 \\
\hline $5 \mathrm{LOV} 517$ & 40 & 39.22 & 119 & 37.76 & 3940 & 979837.8 & -19.0 & -153.4 & 0.9 & 2.4 & -152.3 & 2.8 \\
\hline $5 \mathrm{LOV} 518$ & 40 & 38.89 & 119 & 38.09 & 3993 & 979835.7 & -15.7 & -151.9 & 0.6 & 1.9 & -151.3 & \\
\hline 5LOV519 & 40 & 38.86 & 119 & 38.33 & 4118 & 979830.7 & -8.9 & -149.3 & 0.8 & 1.9 & -148.8 & 6.2 \\
\hline 05LOV520 & 40 & 38.86 & 119 & 38.58 & 4206 & 23.4 & -7.9 & -151.3 & 0.9 & 1.9 & -150.7 & \\
\hline $5 \mathrm{LOV} 521$ & 40 & 37.33 & 119 & 38.94 & 4152 & 979824.6 & -9.4 & -151.0 & 0.9 & 1.3 & -151.0 & 3.4 \\
\hline $5 \mathrm{LOV} 522$ & 40 & 36.97 & 119 & 42.91 & 944 & 979823.3 & -29.8 & -164.4 & 0.1 & 0.6 & -165.1 & -10.2 \\
\hline 05LOV523 & 40 & 37.31 & 119 & 43.13 & 3973 & 979822.4 & -28.5 & -164.0 & 0.1 & 0.6 & -164.6 & -9.6 \\
\hline 05LOV524 & 40 & 37.60 & 119 & 42.84 & 4028 & 979819.9 & -26.2 & -163.6 & 0.2 & 0.7 & -164.1 & -9.1 \\
\hline $05 \mathrm{LO}$ & 40 & 37.94 & 119 & 42.54 & 4150 & 979 & -21.0 & -162.5 & 0.2 & 0.9 & -163.0 & -7.8 \\
\hline $05 \mathrm{LO}$ & 40 & 38.37 & 119 & 42.24 & 4385 & 5.7 & -8.0 & -157.6 & 0.2 & 0.9 & -158.0 & -2.7 \\
\hline 05LOV527 & 40 & 38.82 & 119 & 41.80 & 1503 & 979801.4 & -1.9 & -155.5 & 0.3 & 1.2 & -155.6 & -0.3 \\
\hline 05LOV528 & 40 & 39.10 & 119 & 41.52 & 4564 & 979798.8 & 0.9 & -154.8 & 0.6 & 1.6 & -154.6 & 0.8 \\
\hline 05LOV529 & 40 & 21.67 & 119 & 49.38 & 3844 & 814.9 & -24.9 & -156.0 & 0.2 & 0.8 & -156.4 & -5.0 \\
\hline 05LOV530 & 40 & 21.72 & 119 & 48.84 & 3844 & 979 & -28.6 & -159.6 & 0.0 & 0.5 & -160.4 & -9.0 \\
\hline V531 & 40 & 21.72 & 119 & 48.30 & 3845 & 10.6 & -29.1 & -160.3 & 0.0 & 0.3 & -161.2 & -9.8 \\
\hline 05LOV532 & 40 & 21.72 & 119 & 47.74 & 3843 & & -32.0 & -163.0 & 0.0 & 0.2 & -164.0 & -12.7 \\
\hline 05LOV533 & 40 & 21.72 & 119 & 47.16 & 3844 & 979804.2 & -35.6 & -166.7 & 0.0 & 0.2 & -167.7 & -16.4 \\
\hline 05LOV534 & 40 & 21.72 & 119 & 46.55 & 3846 & 979802.7 & -36.9 & -168.1 & 0.0 & 0.2 & -169.1 & -17.8 \\
\hline 05LOV535 & 40 & 21.29 & 119 & 46.56 & 3845 & 979802.6 & -36.4 & -167.6 & 0.0 & 0.2 & -168.6 & -17.4 \\
\hline $05 \mathrm{LC}$ & 40 & 20.86 & 119 & 46.56 & 3845 & 979 & -36.4 & -167.5 & 0.0 & 0.2 & -168.6 & -17.4 \\
\hline 05LOV537 & 40 & 20.86 & 119 & 47.13 & 3866 & 03.0 & -33.5 & -165.3 & 0.0 & 0.2 & -166.4 & -15.1 \\
\hline 05LOV538 & 40 & 20.85 & 119 & 47.73 & 3845 & 979806.2 & -32.2 & -163.3 & 0.0 & 0.3 & -164.3 & -13.0 \\
\hline 05LOV539 & 40 & 20.86 & 119 & 48.26 & 3843 & 979808.1 & -30.5 & -161.6 & 0.0 & 0.3 & -162.5 & -11.2 \\
\hline 05LOV540 & 40 & 20.86 & 119 & 48.84 & 843 & 13.2 & -25.5 & -156.5 & 0.1 & 0.5 & -157.2 & -5.9 \\
\hline 05LOV541 & 40 & 20.20 & 119 & 48.06 & 3847 & 979805.3 & -31.9 & -163.2 & 0.0 & 0.3 & -164.0 & -12.8 \\
\hline 05LOV542 & 40 & 20.20 & 119 & 47.48 & 3851 & 979803.3 & -33.6 & -164.9 & 0.0 & 0.2 & -165.9 & -14.8 \\
\hline 05LOV543 & 40 & 20.19 & 119 & 46.89 & 3847 & 979802.1 & -35.1 & -166.3 & 0.0 & 0.2 & -167.3 & -16.2 \\
\hline 05LOV544 & 40 & 20.20 & 119 & 48.06 & 3847 & 979805.1 & -32.1 & -163.4 & 0.0 & 0.3 & -164.2 & -13.0 \\
\hline 05LOV545 & 40 & 17.04 & 119 & 43.99 & 3858 & 979804.2 & -27.2 & -158.8 & 0.0 & 0.3 & -159.7 & -8.8 \\
\hline 05LOV546 & 40 & 17.47 & 119 & 43.97 & 3855 & 979805.1 & -27.4 & -158.9 & 0.0 & 0.3 & -159.8 & -8.9 \\
\hline 05LOV547 & 40 & 17.91 & 119 & 43.97 & 3852 & 979807.0 & -26.4 & -157.8 & 0.0 & 0.3 & -158.8 & -7.8 \\
\hline 05LOV548 & 40 & 18.34 & 119 & 43.96 & 3853 & 979808.7 & -25.2 & -156.7 & 0.0 & 0.3 & -157.6 & -6.6 \\
\hline 05LOV549 & 40 & 18.78 & 119 & 43.96 & 3857 & 979809.9 & -24.3 & -155.9 & 0.0 & 0.3 & -156.8 & -5.8 \\
\hline GERL & 40 & 39.09 & 119 & 21.17 & 3934 & 979815.4 & -41.8 & -176.0 & 0.0 & 0.5 & -176.8 & -23.6 \\
\hline GRAVEL & 40 & 39.73 & 119 & 22.16 & 3978 & & -26.1 & -161.7 & 0.5 & 1.1 & -161.9 & -8.5 \\
\hline HERLONG & 40 & 8.67 & 120 & 7.90 & 4094 & 979757.1 & -39.8 & -179.4 & 0.0 & 0.4 & -180.3 & -28.1 \\
\hline NVGASPIP & 40 & 23.16 & 119 & 49.71 & 3902 & 979816.1 & -20.4 & -153.4 & 0.3 & 1.4 & -153.3 & -1 \\
\hline SOSCD & 40 & 12.61 & 119 & 49.28 & 4031 & 979796.0 & -12.7 & -150.2 & 0.0 & 0.5 & -150.9 & \\
\hline
\end{tabular}

